\title{
Ten-year optical monitoring of PKS 0735+178: historical comparison, multiband behavior, and variability timescales
}

\author{
S. Ciprini ${ }^{1,2,3}$, L. O. Takalo ${ }^{1}$, G. Tosti ${ }^{2,3}$, C. M. Raiteri ${ }^{4}$, M. Fiorucci ${ }^{2}$, M. Villata $^{4}$, \\ G. Nucciarelli ${ }^{2}$, L. Lanteri ${ }^{4}$, K. Nilsson ${ }^{1}$, and J. A. Ros ${ }^{5}$
}

1 Tuorla Astronomical Observatory, University of Turku, Väisäläntie 20, 21500 Piikkiö, Finland e-mail: stefano.ciprini@utu.fi

2 Physics Department and Astronomical Observatory, University of Perugia, via A. Pascoli, 06123 Perugia, Italy

INFN Perugia Section, via A. Pascoli, 06123 Perugia, Italy

4 INAF, Torino Astronomical Observatory, via Osservatorio 20, 10025 Pino Torinese, Torino, Italy

5 Agrupación Astronómica de Sabadell, Apartado de Correos 50, PO Box 50, 08200 Sabadell, Barcelona, Spain

Received 5 January 2005 / Accepted 28 October 2006

ABSTRACT

\begin{abstract}
Aims. New data and results on the optical behavior of the prominent blazar PKS 0735+178 (also known as OI 158, S3 0735+17, DA 237, 1ES 0735+178, 3EG J0737+1721) are presented, through the most continuous BVRI data available in the period 1994-2004 (about 500 nights of observations). In addition, the whole historical light curve, and a new photometric calibration of comparison stars in the field of this source are reported.

Methods. Several methods for time series analysis of sparse data sets are developed, adapted, and applied to the reconstructed historical light curve and to each observing season of our unpublished optical database on PKS $0735+178$. Optical spectral indexes are calculated from the multi-band observations and studied on long-term (years) durations as well. For the first time in this source, variability modes, characteristic timescales, and the signal power spectrum are explored and identified over 3 decades in time with sufficient statistics. The novel investigation of mid-term optical scales (days, weeks), could be also applied and compared to blazar gamma-ray light curves that will be provided, on the same timescales, by the forthcoming GLAST observatory.

Results. In the last 10 years the optical emission of PKS $0735+178$ exhibited a rather achromatic behavior and a variability mode resembling the shot-noise. The source was at an intermediate or low brightness level, showing a mild flaring activity and a superimposition/succession of rapid and slower flares, without extraordinary and isolated outbursts, but, at any rate, characterized by one major active phase in 2001. Several mid-term scales of variability were found, the more common falling into duration intervals of about 27-28 days, 50-56 days and 76-79 days. Rapid variability in the historical light curve appears to be modulated by a general, slower, and rather oscillating temporal trend, where typical amplitudes of about 4.5, 8.5, and 11-13 years can be identified. This spectral and temporal analysis, accompanying our data publication, suggests the occurrence of distinctive signatures at mid-term durations that can likely be of transitory nature. On the other hand the possible pseudo-cyclical or multi-component modulations at long times could be more stable, recurrent and correlated to the bimodal radio flux behavior and the twisted radio structure observed over several years in this blazar.
\end{abstract}

Key words. BL Lacertae objects: individual: PKS 0735+178 - BL Lacertae objects: general - galaxies: active - galaxies: photometry - methods: statistical

\section{Introduction}

The rapid and violent optical variability is one of the defining properties of blazars, and variability studies are important in understanding the physics of AGN in general. Characteristic timescales, fluctuation modes, flare shapes and amplitudes, duty cycles and spectral changes, correlations, and temporal lags between variations in different spectral bands, provide crucial information on the nature, structure, and location of the emission components and on their interdependencies. In particular, the so-called low/intermediate-frequency peaked BL Lac objects (LBL/IBL) have the peak of the synchrotron emission around infrared and optical wavelengths and commonly show largeamplitude flares characterized by prominent flux variations in a wide range of temporal scales. The rapid optical variations of LBL and IBL are also systematically larger and with shorter duty cycles than those of the high energy peaked BL Lac objects (HBL). Hence a multi-band, possibly well sampled and extended optical monitoring is an important and subsidiary element of the standard multiwavelength (MW) analysis. MW observing campaigns provide, more or less, short snapshots of the targets, lacking of information about their mid-/long-term evolution. Even if the optical band has a narrow spectral extension, it can yield useful information about the synchrotron emission peak and possible disk/host-galaxy contributions. Moreover, long-term (historical) records of blazar variability are available at optical wavelengths for several bright objects, although data collected in the past are rather sparse. Small-size and dedicated (possibly automatic) telescopes, in conjunction with international consortiums, have recently increased the amount of photometric data, sometimes with a fair continuous sampling during specific observing campaigns.

In this paper we present more than 10 years (Feb. 1993Feb. 2004) of monitoring data of the blazar PKS $0735+178$ (1332 photometric points in four BVRI Johnson-Cousins filters, obtained during about 500 observing nights). Our effort represents the best optical monitoring available for this object, 
regarding continuous and long-term coverage. This optical program allowed us to study colors and the continuum spectrum (mainly in VRI bands) and, for the first time, enabled us to study mid-term scales (days, weeks), over an extended data set. These timescales were almost unexplored in blazars due to the irregular/poor sampling and the low statistics in the optical regime. Time series analysis accompanying our data publication is performed for both our observations and the historical light curve (1906-2004), while a new photometric calibration of comparison stars in the field of the source is also reported, as useful reference for future optical observations and monitoring. In the historical light curve there are obvious differences in data quality, accuracy and sampling over time, that can yield biases, noise and spurious/fake signatures. However, we remark that the last 33-years' portion (1970-2004) of the historical light curve holds a sufficiently regular sampling to allow meaningful statistical results on long-term intervals, too. Data binning when needed, the employment and comparison of 7 different temporal analysis methods suitable for an unevenly sampled (sparse) data set, and the calculation of the power spectrum given by the gaps, ensure that we have determined and reported only real and intrinsic time signatures. We note finally that the main aim of our paper was to investigate the variability behavior on such intermediate scales through our 10-year observations. Data published in this work were obtained by 4 optical observatories: the Perugia University Observatory (Italy), the INAF Torino Observatory (Italy), the Tuorla Observatory (Finland), and the Sabadell Observatory (Spain). Perugia, Torino, and Sabadell data on PKS 0735+178 are unpublished, while part of the Tuorla data were already published in Katajainen et al. (2000). Optical data from Qian \& Tao (2004) have been also added to partly improve the sampling.

The paper is organized as follows: in Sect. 2 we briefly review the optical knowledge of PKS 0735+178, while in Sect. 3 we mention the observing and data reduction techniques. A new photometric calibration of comparison stars in the field of the source is presented in Sect. 4, and the BVRI light curves collected during our monitoring are showed in Sect. 5. The reconstructed historical light curve is described in Sect. 6, while in Sect. 7 the analysis of the multi-band behavior is reported computing the optical spectral indexes. A joint temporal analysis of our data and the historical light curve is performed in Sect. 8, and summary and conclusions are outlined in Sect. 9.

\section{Optical properties of PKS $0735+178$}

The radio object PKS $0735+178$, belonging to the Parkes catalog (other most used names are: PKS 0735+17, S3 0735+17, OI 158, DA 237, VRO 17.07.02, PG 0735+17, RGB J0738+177, 1Jy 0735+17, RX J0738.1+1742, 3EG J0737+1721), was identified with an optical point source by Blake (1970). Afterwards it was classified as a classical BL Lac object in Carswell et al. (1974). This source is optically bright, highly variable, and both radio (Kühr et al. 1981) and X-ray selected (Elvis et al. 1992). PKS $0735+178$ has been extensively studied in the radio regime. The radio flux appear to vary quite slowly with some outbursts (Bååth \& Zhang 1991; Teräsranta et al. 1992; Aller et al. 1999; Teräsranta et al. 2004), but there is not any evidence of a radiooptical correlation (Clements et al. 1995; Hanski et al. 2002) or periodicity (Ciaramella et al. 2004). Early radio observations of PKS $0735+178$ showed a peculiar spectrum soon interpreted as the superposition of incoherent synchrotron radiation emitted by distinct and homogeneous radio components, conspiring to add up to an overall very flat shape (the source was indeed

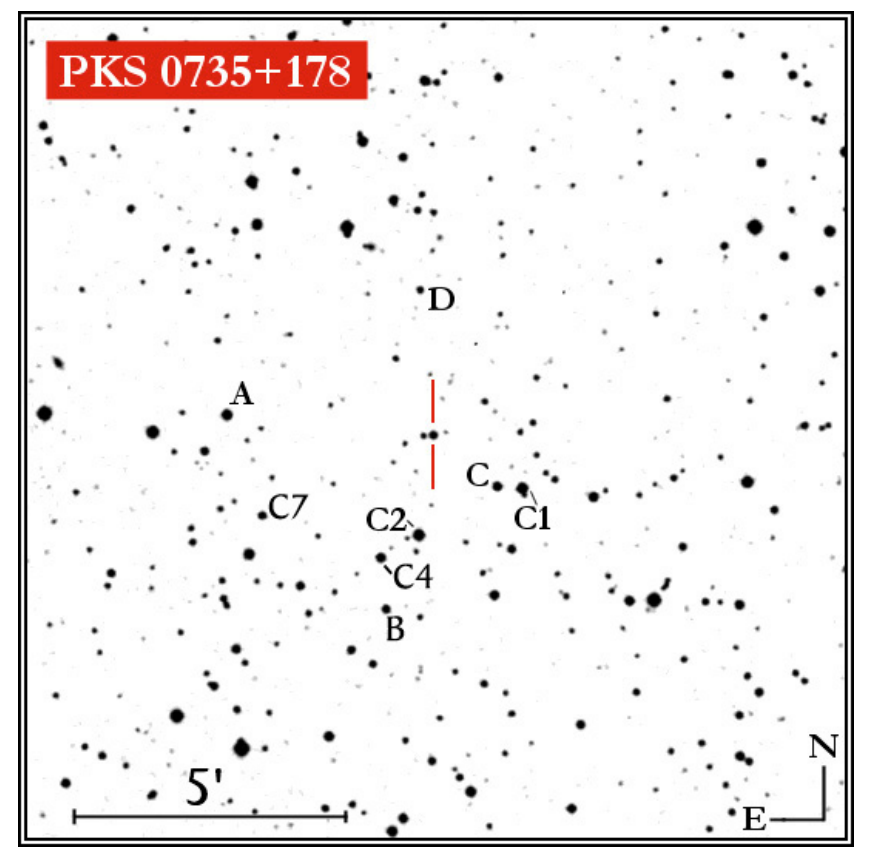

Fig. 1. Finding chart with the optical comparison stars identified in a field of $15^{\prime} \times 15^{\prime}$ centered on PKS $0735+178$ (within the double bar). The field is elaborated from a frame of the Digitized Sky Survey. The new photometric VRI calibration of the stars C1, C, D, C2, C4, C7, and A is reported in Table 1.

nicknamed the "Cosmic Conspiracy", Marscher 1977, 1980; Cotton et al. 1980). Several moving components and an unusual, complex morphology characterized by a twisted jet were observed in VLBA/VLBI radio imaging (see, e.g., Perlman \& Stocke 1994; Gabuzda et al. 1994, 2001; Gómez et al. 2001; Homan et al. 2002; Ojha et al. 2004; Kellermann et al. 2004). PKS $0735+178$ has one of the most bent radio jets among AGN observed at milliarcsecond (mas) scales. A bimodal scenario in which periods of enhanced activity with ejection of superluminal components are followed by epochs of low activity with a highly twisted jet geometry was suggested (Gómez et al. 2001; Agudo et al. 2006). PKS $0735+178$ is also a X-ray and gamma-ray (EGRET, 3EG J0737+1721) emitting blazar (see, e.g., Kubo et al. 1998; Hartman et al. 1999). The spectral energy distribution (SED) evinced PKS $0735+178$ as a low or intermediate-energy peaked BL Lac object (LBL/IBL), where the IR-UV synchrotron continuum dominates the total observed power. The very low X-ray variability with respect to the high optical-IR variations, supported the idea that X-rays are produced by inverse Compton mechanism in some mas radio components (Bregman et al. 1984; Madejski \& Schwartz 1988). The $\gamma$-ray flux of this blazar likewise appeared not strongly variable (Nolan et al. 2003).

The optical spectrum of PKS $0735+178$ shows an absorption line due to an intervening system at $3980 \AA$ that, if identified with $\mathrm{Mg}$-II, provides a lower redshift limit of $z>0.424$ (Carswell et al. 1974; Burbidge \& Hewitt 1987; Falomo \& Ulrich 2000; Rector \& Stocke 2001). A strong Lyman-alpha absorption line has also been detected by the IUE satellite at the same redshift (Bregman et al. 1981). This absorption was not identified in deep optical imaging, even if a very faint emission was detected about 3.0-3.5" NE/E (projected distance $22-25 \mathrm{kpc}$ at $z=0.424$ ) from the object (Falomo \& Ulrich 2000; 
Table 1. A new optical photometric calibration for field of PKS 0735+178, with $V R_{\mathrm{c}} I_{\mathrm{c}}$ Johnson-Cousins sequences of comparison stars C1, C, D, C2, C4, C7, and A (see Fig. 1).

\begin{tabular}{|c|c|c|c|c|c|}
\hline \multicolumn{6}{|c|}{ Photometric sequences for PKS $0735+178$ comparison stars } \\
\hline Star & $\begin{array}{c}\text { RA } \\
(\mathrm{J} 2000.0)\end{array}$ & $\begin{array}{c}\text { Dec } \\
(\mathrm{J} 2000.0)\end{array}$ & $\begin{array}{c}V \\
{[\mathrm{mag}]}\end{array}$ & $\begin{array}{c}R_{\mathrm{c}} \\
{[\mathrm{mag}]}\end{array}$ & $\begin{array}{c}I_{\mathrm{c}} \\
{[\mathrm{mag}]}\end{array}$ \\
\hline 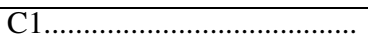 & 073800.5 & +174119.9 & $13.26 \pm 0.04$ & $12.89 \pm 0.04$ & $12.57 \pm 0.04$ \\
\hline С & 073802.4 & +174122.2 & $14.45 \pm 0.04$ & $13.85 \pm 0.04$ & $13.32 \pm 0.04$ \\
\hline D.... & 073808.3 & +174459.7 & $15.90 \pm 0.05$ & $15.49 \pm 0.05$ & $15.12 \pm 0.06$ \\
\hline $\mathrm{C} 2 .$. & 073808.5 & +174029.2 & $13.31 \pm 0.04$ & $12.79 \pm 0.04$ & $12.32 \pm 0.04$ \\
\hline .......... & 073811.6 & +174004.4 & $14.17 \pm 0.05$ & $13.80 \pm 0.04$ & $13.48 \pm 0.04$ \\
\hline $\mathrm{C} 7 \ldots$ & 073820.7 & +174051.2 & $15.01 \pm 0.06$ & $14.70 \pm 0.06$ & $14.37 \pm 0.05$ \\
\hline A & 073823.4 & +174243.0 & $13.40 \pm 0.05$ & $13.10 \pm 0.05$ & $12.82 \pm 0.05$ \\
\hline
\end{tabular}

Pursimo et al. 2002). The host galaxy of PKS $0735+178$ remains unresolved in optical imaging (Scarpa et al. 2000; Falomo \& Ulrich 2000; Pursimo et al. 2002), but the source has two well-resolved companion galaxies. The galaxy at $7^{\prime \prime} \mathrm{NW}$, declared distorted by interaction with PKS 0735+178 (Hutchings, Johnson, \& Pyke 1988), does not show marks of interaction in more recent and higher resolution images, and a redshift of $z=0.645$ was obtained for it (Stickel et al. 1993; Scarpa et al. 2000; Falomo \& Ulrich 2000). In addition, a brighter galaxy located at 8.1" SE cannot be the absorber due to the great projected distance from our blazar. A third, very faint, elongated structure at 3-3.5" NE was detected as well (Pursimo et al. 1999; Falomo \& Ulrich 2000), and this could be related to the intervening absorption at $z=0.424$. The lower limit for the redshift $z>0.5 \mathrm{ob}-$ tained assuming typical properties for the host (Falomo \& Ulrich 2000) is consistent with the limit derived from intervening absorption.

When combined with our data the historical optical curve of PKS 0735+178, starting from JD 2417 233, i.e., Jan. 22, 1906 (Fan et al. 1997), spans almost 100 years. Optical variations are often of larger amplitudes than the infrared one (Fan \& Lin 2000). Correlations between the spectral index and the optical brightness were observed (Sitko \& Sitko 1991; Lin \& Fan 1998), alongside a spectral flattening (blueing) with the source brightening (Brown et al. 1989; Lin \& Fan 1998). On the other hand this type of correlation appears to be weak (Gu et al. 2006) or opposite (i.e., spectral steepening, reddening; Ghosh et al. 2000) in other multiband observations. The largest optical variations registered are on the order of 3-4 mag (Pollock et al. 1979; Fan et al. 1997) at long (years) ranges. Some intra-day (IDV) and inter-day variations until $0.5 \mathrm{mag}$ were reported in the optical history of this blazar (Xie et al. 1992; Fan et al. 1997; Massaro et al. 1995; Zhang et al. 2004). Over the period 1995-1997, the optical IDV and microvariations were rare and with a small amplitude (Bai et al. 1999), while no clear evidence was found in more recent observations (Sagar et al. 2004). Several possible recurrent and pure periodical components were claimed, with values of 1.2, 4.8 years (Smith et al. 1988; Webb et al. 1988; Smith \& Nair 1995); 14.2, 28.7 years (Fan et al. 1997); and 8.6, 13.8, $19.8,37.8$ years (Qian \& Tao 2004), even if we remark that such scales are derived by different data sets and different epochs. The long-term analysis performed with the Jurkevich's method on a more complete data set (Fan et al. 1997; Qian \& Tao 2004) postulated a main periodical component of about 13.8-14.2 years, but our temporal analysis (Sect. 8) suggests other and shorter long-term signatures.

PKS $0735+178$ also has a relatively high degree of optical polarization showing very different levels covering the whole range from about $1 \%$ up to $30 \%$ (see, e.g., Mead et al. 1990; Takalo 1991; Takalo et al. 1992; Valtaoja et al. 1991, 1993;
Tommasi et al. 2001). Only a modest variability of this optical polarization was observed on inter/intra night durations, which was interpreted as being owed to substructures of different polarization and variable intensity in the jet. A preferred polarization level over few years (Tommasi et al. 2001) could indicate quiescence and stability in the underlying jet structure.

\section{Observations and data reduction}

Photometric observations were carried out with four telescopes. The Newtonian f/5, 0.4 m, Automatic Imaging Telescope (AIT) of the Perugia University Observatory ${ }^{1}$, Italy (451 meters above sea level, a.s.1.), a robotic telescope equipped with a $192 \times$ 165 pixel CCD array, thermoelectrically cooled with Peltier elements (Tosti et al. 1996). The REOSC f/10, $1.05 \mathrm{~m}$, astrometric reflector of the Torino Observatory ${ }^{2}$, Italy (622 meters a.s.1.), mounting a $1242 \times 1152$ pixel CCD array, cooled with liquid nitrogen and giving an image scale of $0.467^{\prime \prime}$ per pixel. The Dall-Kirkham f/8.45, $1.03 \mathrm{~m}$ reflector of the Turku University Tuorla Observatory ${ }^{3}$, Finland (60 meter a.s.l.), equipped with a $1530 \times 1020$ pixel CCD camera, which is thermoelectrically cooled. The Newtonian $0.5 \mathrm{~m}$ telescope of the Sabadell Observatory ${ }^{4}$, Spain used in two interchangeable configurations (Newton at f/4, and Cassegrain-Relay at f/15), and equipped with a $512 \times 512$ FLI CM-9 CCD array. The Perugia and Torino telescopes were provided with standard $B V$ (Johnson) and $R_{\mathrm{c}} I_{\mathrm{c}}$ (Cousins) filters (Bessell 1979; Fiorucci \& Munari 2003; Bessell 2005). The Tuorla $1 \mathrm{~m}$ telescope, and the Sabadell telescope were equipped with $\mathrm{V}$ and $R_{\mathrm{c}}$ filters.

All the observatories took CCD frames and performed a first automatic data reduction using standard methods, to correct each raw image (for dark and bias background signals where needed) and to flat fielding, to recognize the field stars, and to derive instrumental magnitudes via aperture photometry (Perugia and Tuorla) or circular Gaussian fitting (Torino). The single frames are then inspected to evaluate the quality of the image, the reliability of the data, and to search for spurious interferences. Comparison among data obtained with these different telescopes on the same night reveals a good agreement, and no detectable offset is found. The matching with data taken in simultaneous epochs at Shanghai Observatory (Qian \& Tao 2004) showed a good agreement too (see Fig. 3). The precision level in the light curve of PKS 0735 assembled in this way is enough for a variability analysis performed on intermediate and longterm timescales. Moreover the time series analysis of shorter

\footnotetext{
1 http://astro.fisica.unipg.it

2 http://WWW.to.astro.it

3 http://www .astro.utu. fi

4 http://wWw . astrosabadell.org
} 


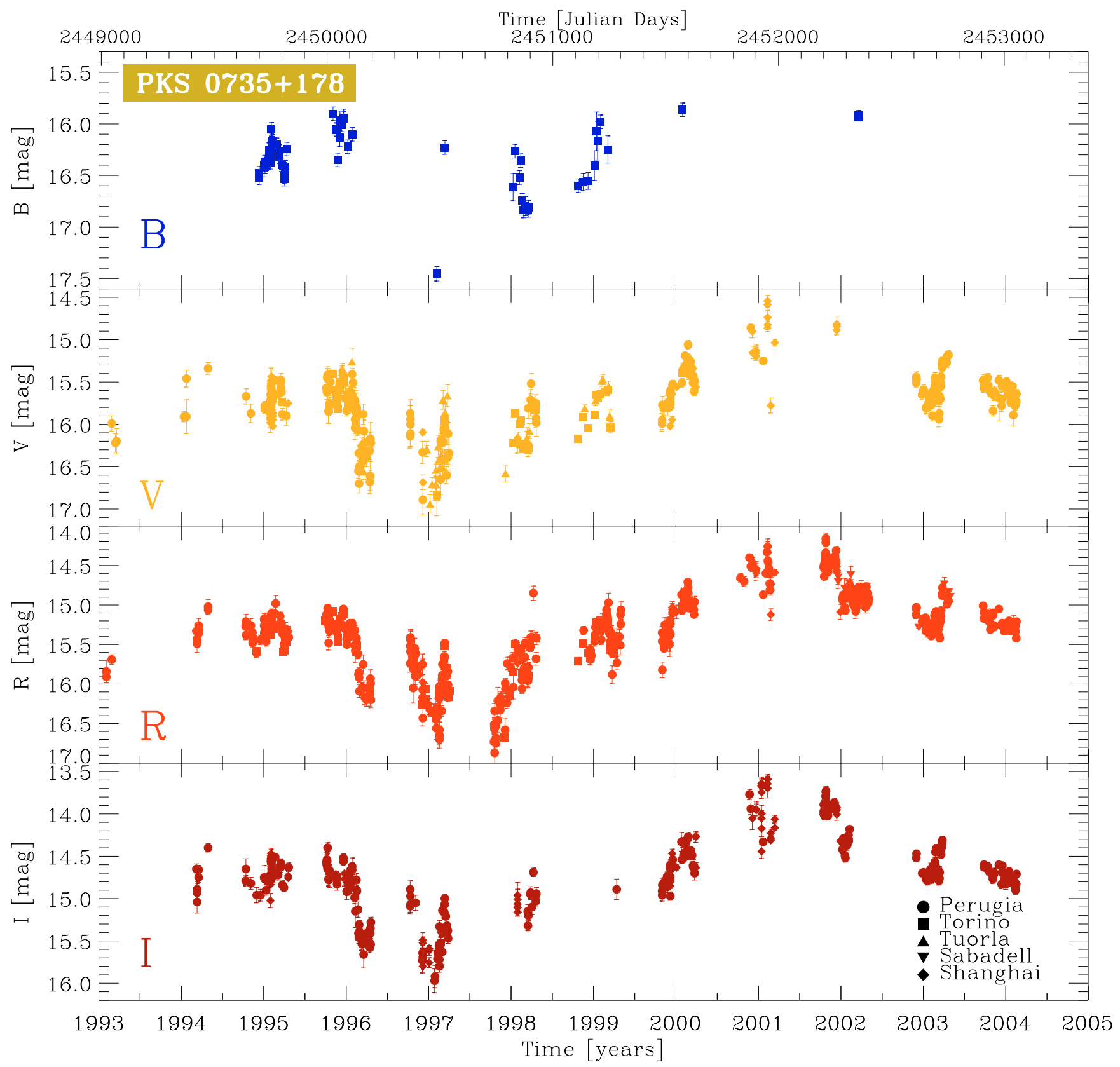

Fig. 2. BVRI magnitude light curves of PKS 0735+178 from 1993 to the beginning of 2004. Data came from our 10-year observing monitoring.

timescales ( $<200$ days) is performed in each single observing season using $R$-band data, which were mainly obtained by 1 telescope (see Table 2).

\section{Comparison stars' photometry}

Calculation of the source magnitude is easily obtained by differential photometry with respect to comparison stars in the same field of the object. The discussion of the adopted comparison star sequence is crucial for analysis of optical data obtained during blazar monitoring observations, as the photometric sequence affects data quality and reliability. To obtain a dependable photometric sequence for PKS $0735+178$, we selected a set of non-variable stars with brightness comparable to the object and different colors (see the finding chart in Fig. 1). Photometric calibrations of these stars were derived from 13 optimal photometric nights between 1994 and 1996 at the Perugia University
Observatory using Landolt standards. The stability of the sequence for the stars C1, C, D, C2, C4, was well tested and verified during data reduction of the overall database (for stars $\mathrm{A}$ and $\mathrm{C} 7$ there are fewer measurements because of the small FOV of the instrument). Our new photometric sequence is presented in Table 1 , showing the $V$ (Johnson), and $R_{\mathrm{c}}, I_{\mathrm{c}}$ (Cousins) photometric values and was adopted in this work. A, C, and D stars also belong to the photometric sequence calibrated by Smith et al. (1985), to the photometric sequence published in Wing (1973, here with a B sequence too), and the sequence that appeared in Veron \& Veron (1975, A and D stars only). Stars C2, C4, and C7 also belong to the photometric sequence calibrated by McGimsey et al. (1976, named there 1,2,3), while for the $\mathrm{C} 1$ star the calibration is totally new (Fig. 1 and Table 1). Magnitudes of common stars are roughly in agreement within the uncertainties. In particular the determinations of McGimsey et al. (1976) are photoelectric in the $U B V$ Johnson system, thus 
well similar to our calibrations in the $B$ and $V$ bands using a CCD detector. Discrepancies are small if the specifications of Bessell (1990) are respected and determined (Fiorucci \& Munari 2003). The $V R_{\mathrm{c}} I_{\mathrm{c}}$ sequence reported in Table 1 , joined with the $U, B$ values reported by Smith et al. (1985), is suggested for future optical observations and monitoring of this blazar.

This new photometric calibration of the PKS 0735+178 field, (suitable also for telescopes with small FOV), is slightly more extended and accurate with respect to the past calibrations. Table 1 reports the $V, R_{\mathrm{c}}, I_{\mathrm{c}}$ values for seven comparison stars, while the sequence for the star denoted with $\mathrm{C} 1$ is completely new. In each photometric night, standard Landolt stars were observed at different airmasses, and the calibration line as a function of the airmass was constructed (neglecting the color corrections always being smaller than the instrumental errors). The standard magnitudes of such stars were derived (with error equal to the quadrature sum of the linear regression error and the instrumental error on the single star). The typical error for each night and each star is between 0.03 and $0.1 \mathrm{mag}$ (depending on the luminosity of the star and the atmospheric conditions). Data shown in Table 1 are the result of weighted averages on the values of each night (weight equal to $1 / \sigma^{2}$ ), whereas the error estimation is equal to the standard deviation weighted on the averages. This uncertain is higher than the standard deviation on each single night because some systematic errors, were different on each night. We chose to report a reliable calibration with reliable errors in Table 1 with respect to the more accurate but more doubtful smoothed values. Color transformations to comparison stars were not applied because from the analysis of Landolt stars it was not possible to separate this effect from the instrumental statistical errors given mainly by the effective limits of the Perugia instrument and site. Then again the photometric system of the Perugia telescope was developed to follow at best the standard Johnson-Cousins system. Finally we note that some of the stars listed in Table 1 are quite red $(V-I$ index ranges from +0.58 to $+1.13 \mathrm{mag}$, suggesting that they have spectral types $\mathrm{F}$, $\mathrm{G}$, possibly K), but these color indexes are similar to the color indexes of PKS $0735+178$ (average $V-I=+0.95 \pm 0.1$, average $B-R=+0.91 \pm 0.09$ ) hence color effects are correspondent, and $B$-band data were obtained only with the larger $1 \mathrm{~m}$ Torino telescope. A detailed description of the observing and data reduction procedures, filter system, software adopted in the calibrations, and comparison with other works can be found in Fiorucci et al. (1998) and Fiorucci \& Tosti (1996).

\section{Optical light curves from 1993 to 2004}

We monitored the BL Lac object PKS $0735+178$ in the four $B, V, R_{c}, I_{c}$ optical bands for more than 10 years, from February 2, 1993 to February 17, 2004 (JD = 2449 021-2453 053). A total of $1332 B V R I$ reduced and validated photometric points were obtained over a period of 4032 days (see Fig. 2). To obtain a more complete light curve, data obtained at the Shanghai Observatory from Qian \& Tao (2004) are added to this figure and to our analysis. Data sets of different observatories are roughly in agreement within the uncertainties (Figs. 2 and 3). In the best sampled band (the $R$-band, analyzed in detail in Sect. 8), 709 photometric points were collected over 12 observing seasons (see Tables 2 and 3) with 459 nights in total having at least one $R$ data point. The last and best sampled 10 observing seasons (from the III to the XII, i.e., from October 1994 to February 2004) have a duration spanning from 144 to 203 days Table 3), an average number of data points per night equal to 1.5 , an average empty gap between subsequent observations of 3 days, and

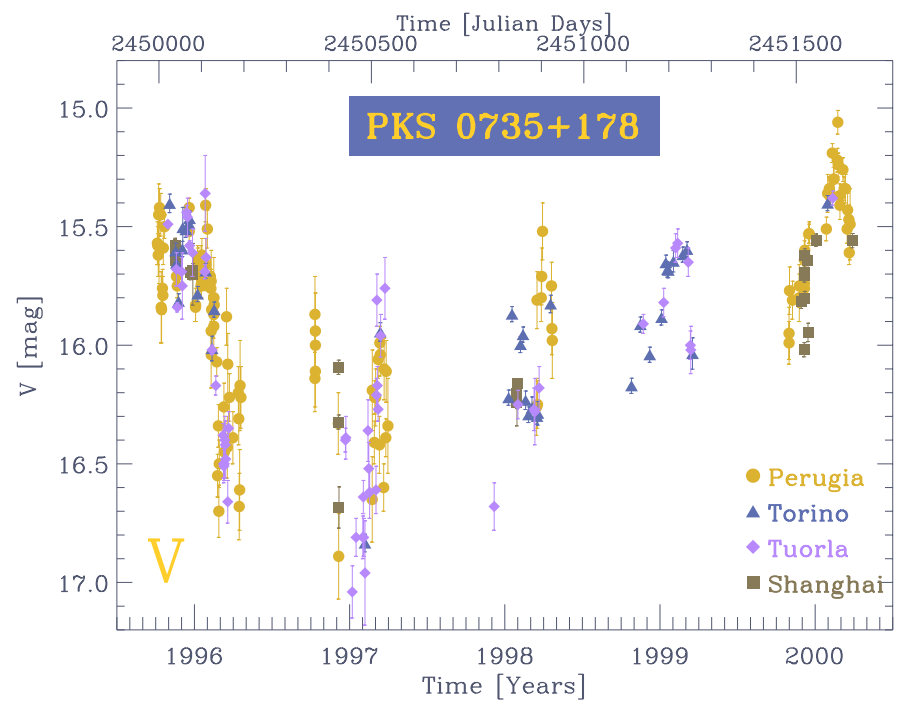

Fig. 3. A portion of the $V$-mag light curve where it is possible to see data from the different observatories in more detail.

an average coverage of nights having data with respect to each season duration of about $27 \%$. Practically speaking such numbers mean that data are not clustered or bunched, and that a regular enough monitoring was performed (when permitted by atmospheric/technical conditions). The priority of our observing program during these years was to perform a constant and possibly uniform optical monitoring. Consequently, for the first time in PKS 0735+178, this allowed us to obtain data suitable for a deep and detailed statistical analysis on days/weeks timescales. The majority of such $R$-filter observations were obtained by only two telescopes (over the $69 \%$ obtained by the Perugia telescope, and a further $21 \%$ by the Torino telescope) having known interinstrumental offsets below $0.1 \mathrm{mag}$ in this band (e.g. Villata et al. 2002; Böttcher et al. 2005). Three examples of such R-band seasonal light curves with the accompanying time series analysis functions are reported in Figs. 10-12.

A direct visual inspection of our 10-year multiband light curve (Fig. 2), shows an average optical brightness placed at a mid or low levels, displaying rapid variability with a moderate flaring but no extraordinary big/isolated outburst $(R>14$ in the whole data set). Luminosity drops/increasing of about 2 magnitudes were common on time intervals smaller than half a year. From the end of 1997, a slow increase of the average brightness was clearly detected (e.g., the $R$-band magnitude never dropped to values higher than 16 from beginning of 1998), while in 2001 a clear brightening phase can be well identified. This moderateoutburst phase can be considered comparable to the other outbursts seen in the optical history of PKS 0735+178 (see Sect. 6).

\section{The historical light curve}

The optical history of PKS $0735+178$ together with our data (Fig. 4) extends over almost one century (from Jan. 22, 1906, JD 2417233, to Feb. 17, 2004, JD 2453053). The older points in the light curve were obtained using plates (mainly from the Landessternwarte Heidelberg-Königstuhl Observatory, Germany, and the Rosemary Hill Observatory, Florida, USA; Zekl et al. 1981; Webb et al. 1988), from which a photographic magnitude $m_{\text {pg }}$ can be extracted and converted in the photometric $B$ magnitude following a semi-empirical correction (see, e.g., Lu 1972; Kidger 1989). More recent data have been obtained 
Table 2. The number of photometric BVRI data points of PKS $0735+178$ obtained by each observatory in the period 1993-2004 and published in this paper. In the bottom panel a summary of the basic information and statistics about our data in each band.

\begin{tabular}{|c|c|c|c|c|c|c|c|c|}
\hline \multicolumn{9}{|c|}{ Data points per observatory } \\
\hline Obs. & $\bar{B}$ & $V$ & $R$ & $I$ & Tot. & \multicolumn{3}{|c|}{ Period } \\
\hline Perugia & 0 & 226 & 490 & 281 & \multirow{2}{*}{997} & \multicolumn{3}{|c|}{ Feb.1993-Feb.2004 } \\
\hline Torino & 75 & 38 & 150 & 0 & & \multicolumn{3}{|c|}{ Dec.1994-Apr.2002 } \\
\hline Tuorla & 0 & 55 & 0 & 0 & 55 & \multicolumn{3}{|c|}{ Oct.1995-Feb.2001 } \\
\hline Sabadell & 0 & 0 & 17 & 0 & 17 & \multicolumn{3}{|c|}{ Dec.2001-Feb.2004 } \\
\hline Shanghai & 0 & 115 & 52 & 138 & 305 & \multicolumn{3}{|c|}{ Jan.1995-Dec.2001 } \\
\hline Total & 75 & 434 & 709 & 419 & \multicolumn{4}{|c|}{1637} \\
\hline \multicolumn{9}{|c|}{ 1993-2004 data statistics } \\
\hline & & & & $B$ & & $V$ & $R$ & $I$ \\
\hline \multicolumn{4}{|c|}{ Total data points } & 75 & & 434 & 709 & 419 \\
\hline \multicolumn{4}{|c|}{ Start date [JD-2 449000$]$} & 698 & & 45 & 21 & 420 \\
\hline \multicolumn{4}{|c|}{ End date [JD-2 449000$]$} & 335 & & 4053 & 4053 & 4053 \\
\hline \multicolumn{4}{|c|}{ Total period $N_{\text {tot }}$ [days] } & 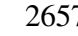 & & 4001 & 4032 & 3633 \\
\hline \multicolumn{4}{|c|}{ Nights with data $N_{\text {on }}$} & 52 & & 297 & 459 & 259 \\
\hline \multicolumn{4}{|c|}{$N_{\text {on }} / N_{\text {tot }}$ fraction } & 0.01 & & 0.074 & 0.171 & 0.071 \\
\hline \multirow{2}{*}{\multicolumn{4}{|c|}{$\begin{array}{l}\text { Mean num. points } \times \text { night } \\
\text { Total mean gap } \Delta t \text { [days] }\end{array}$}} & 1.44 & & 1.46 & 1.51 & 1.62 \\
\hline & & & & 35.9 & & 9.3 & 5. & 8.7 \\
\hline \multicolumn{4}{|c|}{ Longest gap [days] } & 780 & & 352 & 375 & 356 \\
\hline \multicolumn{4}{|c|}{ Average brightness [mag] } & 16.31 & & 15.760 & 15.301 & 14.693 \\
\hline \multicolumn{4}{|c|}{ Max brightness [mag] } & 15.86 & & 14.544 & 14.16 & 13.59 \\
\hline \multicolumn{4}{|c|}{ Min brightness [mag] } & 17.45 & & 16.94 & 16.87 & 15.97 \\
\hline \multicolumn{4}{|c|}{ Variability range $\Delta m[\mathrm{mag}]$} & $1.5 \mathrm{~s}$ & & 2.39 & 2.71 & 2.38 \\
\hline \multicolumn{4}{|c|}{ Absorption coeff. ${ }^{\dagger}[\mathrm{mag}]$} & 0.15 & & 0.117 & 0.094 & 0.068 \\
\hline \multicolumn{4}{|c|}{ Data standard deviation } & 0.25 & & 0.368 & 0.515 & 0.453 \\
\hline \multicolumn{4}{|c|}{ Data skewness } & 1.23 & & 0.386 & 0.329 & 0.155 \\
\hline Data kurto & & & & 3.79 & & 1.087 & 0.019 & 0.400 \\
\hline Max flux [ & & & & 2.21 & & 6.1 & 7.3 & 9.9 \\
\hline Min flux $[\mathrm{r}$ & Jy] & & & 0.5 & & 0.67 & 0.60 & 1.1 \\
\hline
\end{tabular}

$\dagger$ Values for the galactic extinction by NED database (Schlegel et al. 1998).

directly with photoelectric or CCD instruments. The historical data collection was taken directly from Qian \& Tao (2004) with few integrations, and appending the original and derived $B$-magnitudes from our database of unpublished observations, (the derived $B$-band data are estimated from our best sampled $R$-mag data after 1993, using a constant color index with value equal to previous works $B-R=0.993$, Fan et al. 1997; Qian \& Tao 2004). The total historical time series obtained in this way is composed of 1725 final data points. Error bars are not represented for clarity. In general a prudent error estimation (taking into account different offsets, different data quality, systematic errors, and instrumental dispersion), needs to be figured out, before using heterogeneous historical optical light curves for a quantitative analysis. In this specific case the further errors introduced by using a constant conversion index from the $R$ - and $B$-band also has to be counted on. This estimation is difficult without all the original data sets (plates, frames, etc.), but based on experiences a reasonable and prudential upper limit to the amplitude of errors in Fig. 4 might be considered around the value of \pm 0.4 mag.

The largest outbursts or brightening phases (mag $B \lesssim 15$ ) occurred in the period Dec. 1937-Feb. 1941, Apr. 1949-Feb. 1950, around Feb.-May 1977, in the period Oct. 1980-Mar. 1981, and Feb. 2001-Oct. 2001. The brightest outburst was observed around mid of May 1977 (JD 2443277-78), when PKS $0735+178$ reached its historical optical maximum $(B=$ 13.9). A rather humped, swinging, and oscillating longterm trend appears to modulate the rapid variability of
PKS 0735+178. This aspect might suggest a cyclical or intermittent trend, with possible pseudo-periodic or multicomponent oscillations running on long-term ranges.

\section{Optical spectral indexes}

The continuum spectral flux distribution of blazars in the optical range can be analyzed to properly distinguish all the emission components that, together with synchrotron radiation, contribute to the observed spectrum shape. Moreover optical flux variations in blazars are frequently associated with changes in the spectral shape. This can be revealed by analyzing the magnitude color indexes or the flux spectral indexes. In calculating the color indexes and the continuum spectral slopes, we selected the more accurate multi-band data ( 3 filters at least) about PKS $0735+178$, obtained by a single telescope, and coupling frames with a maximum time lag of 20 minutes (to reduce possibly intrinsic/extrinsic, instrumental micro-variations). Since both the host galaxy of PKS $0735+178$ and the feature possibly responsible for the intervening absorption at $z=0.424$ are rather faint (see Sect. 2), it is reasonable to neglect the galaxy color interference and any thermal contribution in the observed continuum optical spectra. The observed magnitudes were transformed into flux densities, corrected by the Galactic absorption (derived by Schlegel et al. 1998, see Table 2 lower panel) for the source (located at moderate Galactic latitude, $b=18.07$ ). The absorption is rather small (i.e., $B-I=0.8 \mathrm{mag}$ ), and accordingly the color correction is little in comparison to the mean $B-I$ value of the source. Fluxes relative to zero-magnitude values are taken from the Johnson-Cousins system calibration presented in Bessell (1979, 1990) and Fiorucci \& Munari (2003).

The optical spectral energy distribution (SED), can be expressed conveniently by a power law $v F_{v} \propto v^{-\alpha+1},(v$ being the frequency of radiation and $\alpha$ the spectral index). In the optical regime the degree of correlation between $\alpha$ and the flux sheds light on the non-thermal emission processes (e.g., synchrotron and inverse-Compton processes) is produced by a population of relativistic electrons in the jet. The degree of correlation between the spectral index $\alpha$ and the flux in various bands, through a least-square linear regression was checked, and values characterized by large errors and bad $\chi^{2}$ were rejected. We found that the spectral index $\alpha$ of PKS $0735+178$ varies between $2.11 \pm 0.26$ to $0.66 \pm 0.20$, with an average value of $1.25 \pm 0.15$ in the last ten years. These values for $\alpha$ are roughly in agreement with the values calculated previously in Fiorucci et al. (2004) using only the Perugia Observatory data set.

In Fig. 5 (upper panel) the temporal behavior of $\alpha$ is represented in comparison with the flux light curve in the bettersampled $R$-band. The long-term variability seems essentially achromatic, and there is no obvious correlation between the light curve and the spectral index, whereas flares and short-term variations can imply spectral changes. This is the same behavior found in BL Lac (Villata et al. 2002, 2004) and in S5 0716+71 (Ghisellini et al. 1997; Raiteri et al. 2003). Unfortunately there is almost no spectral information during the 2001 outburst (lack of $B$ and $V$ data) to check a spectral flattening. The few data suggests a rather constant spectral index. In the same Fig. 5 (middle panel) the scatter plot between $\alpha$ and the flux is reported. Data dispersion is evident, and can be explained as statistical fluctuations due to instrumental and calculation errors and due to intrinsic scattering of $\alpha$ during the more rapid and larger flares. A weak linear correlation is outlined and a general spectral flattening is not detected clearly. Uncorrelated random fluctuations in the emitted flux might introduce a 

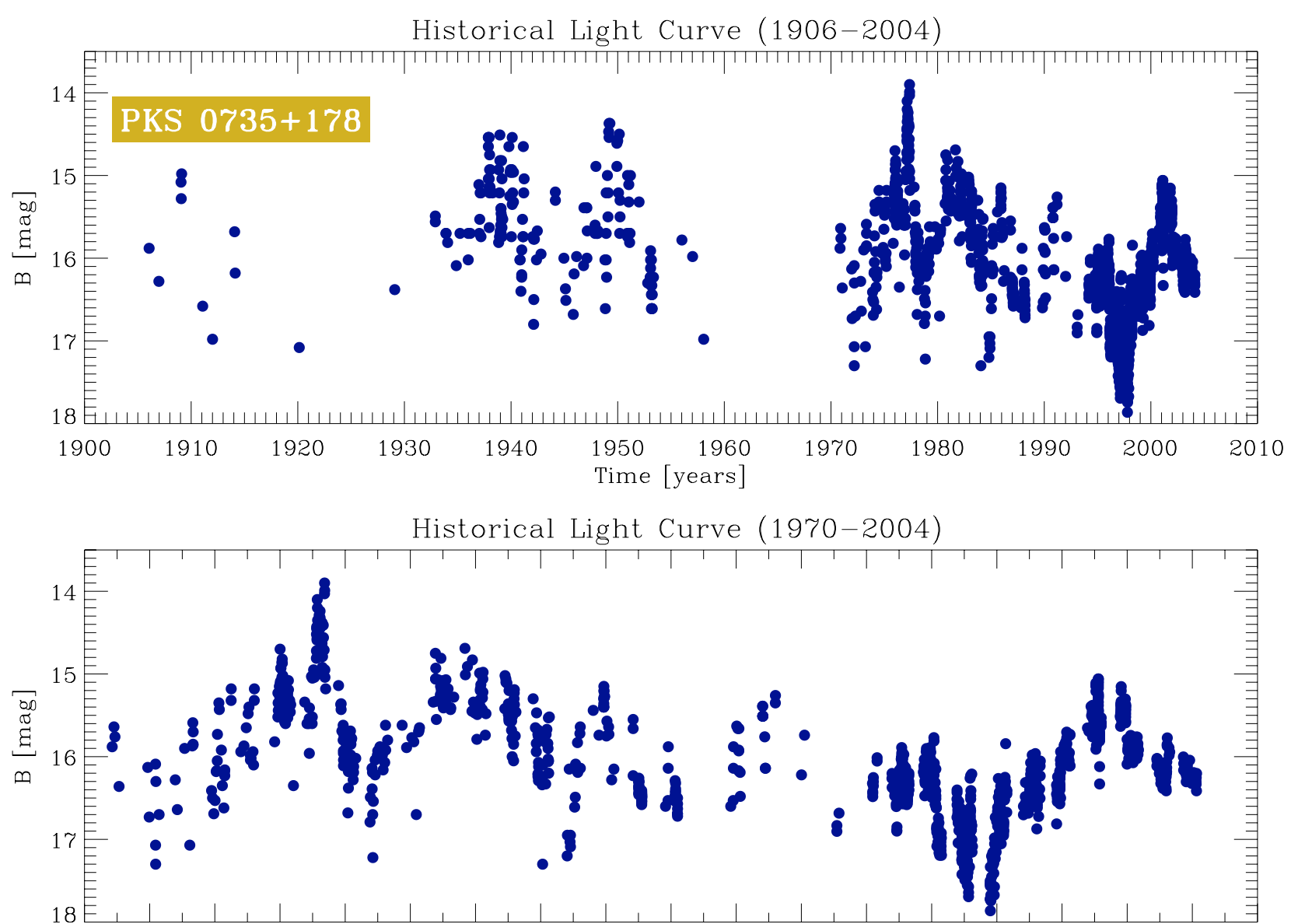

1970197219741976197819801982198419861988199019921994199619982000200220042006 Time [years]

Fig. 4. The historical optical light curve of PKS $0735+178$ in the $B$ band reconstructed by published data in addition to our original $B$ and $R$-derived data points. At least five main outbursts followed by a general humped and rather oscillatory long-term trend can be visually recognized. This behavior is more easily identifiable in the 1970-2004 portion (lower panel), thanks to a higher sampling (lower panel).

statistical bias, due to the spectral index dependence by the flux(Massaro \& Trevese 1996), but values computed for the central frequency (close to the $R$-band, as plotted in Fig. 5), can be considered unbiased and representative of the brightness state. The spectral index $\alpha$ showed consistent variations even when the light curve has rather small variations. On the contrary in the lower panel of Fig. 5 the scatter plot between the fluxes in the $R$ - and $B$-bands shows a well correlated emission as expected (linear correlation coefficient $r_{B-R}=0.90 \pm 0.18$ and slope $0.64 \pm 0.04$ ), without a detectable curvature.

During well-defined and large flares at X-ray bands (especially observed in HBL), the X-ray spectral index versus the flux frequently displays a characteristic loop-like pattern (see, e.g., Georganopoulos \& Marscher 1998; Kataoka et al. 2000; Ravasio et al. 2004). That pattern outlines a hysteresis cycle arising whenever the spectral slope is completely controlled by radiative cooling processes (see, e.g., Kirk et al. 1998; Böttcher $\&$ Chiang 2002). In a few sources this feature was found in the optical regime too (Fiorucci et al. 2004; Ciprini et al. 2004). Consequently we can claim that around and beyond the synchrotron peak frequency, the behavior of the LBL sources during flares in the optical band is scaled in frequency, but possibly very similar to the course of the HBL in X-ray bands.

In our 10-year light curve, PKS 0735+178 showed several moderate-amplitude outbursts, a wider bump of longer duration, and a general flickering or shot-noise type of variability on midterm scales. The evolution of $\alpha$ as a function of the flux is erratic and did not show evident hysteresis loops caused by non-thermal cooling. In Fig. 6 the evolution of $\alpha$ during 3 contiguous observing periods (from January 29, to March 21, 2000) is reported as an example. A rough loop-like behavior is hinted at, meaning that radiative cooling can also dominate the optical SED also during mild flaring activity. Consequently variations at higher frequency band could lead those at the lower frequency bands during both the increasing and decreasing brightness phases, reflecting differences in electron cooling times. The rather limited amplitude of the optical variability in the epochs of Fig. 6, the possible superimposition of different emission processes in the optical band, the under-sampling, and the error propagation in the $\alpha$ calculation can be the main reasons for the lack of welldefined loops in such $\alpha$ vs. flux diagrams. Our data are not sufficient to make a final judgement, and an improved multi-band monitoring and a better data sampling would probably clarify the existence of those patterns also during mild variability in this object.

\section{Temporal variability analysis}

Time series analysis (evolved from both signal-processing engineering and mathematical statistics) provides very useful 


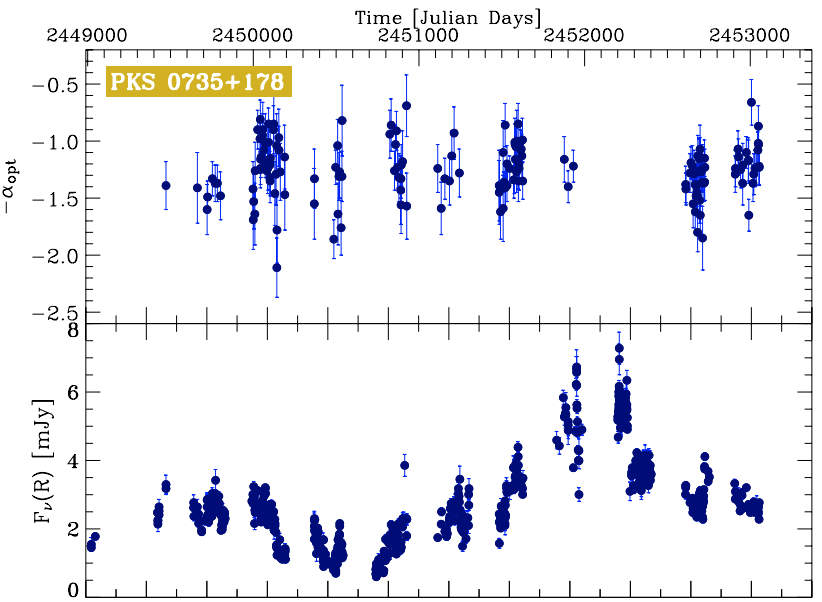

1993199419951996199719981999200020012002200320042005 Time [years]
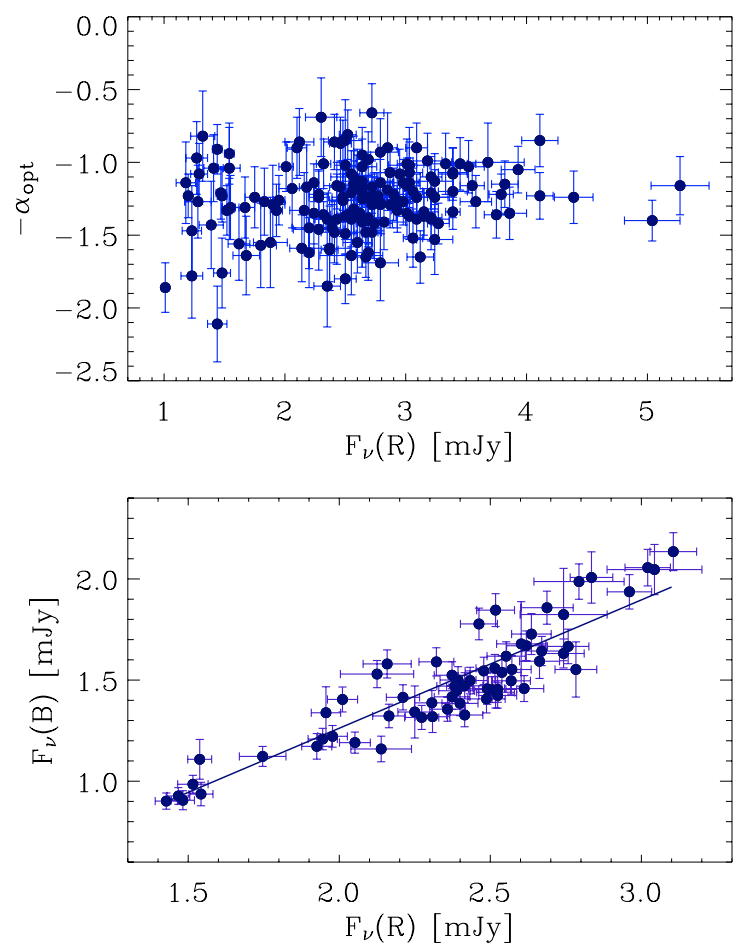

Fig. 5. Upper panel: temporal behavior of the optical spectral index $\alpha$ and the $R$-band flux in PKS 0735+178. Middle panel: dependence of the optical spectral index $\alpha$ on the flux intensity in the $R$-band. Lower panel: the scatter plot between the fluxes in the $R$ - and $B$-bands.

methods to study blazar variability. These methods allow us to explore and extract temporal signatures, structures and characteristic timescales (the powerful scales of variations), duty cycles (the fraction of time spent in an active state), and trends, to determine the dominant fluctuation modes and the power spectrum of the signal. Moreover time series analysis allows us to detect and study auto/cross-correlations, time lags, transient events, periodicity and composite modulations, scaling and coherency, oscillations, beatings and instabilities, intermittence and drifts, dissipation, dumping, long-memory patterns and selfsimilarity, resonance and relaxation processes, random and deterministic features, linear and non-linear processes, and stationary and non-stationary activity, as well as to perform filtering and forecast. The analysis of the flux evolution over time in a blazar, joined with the multiwavelength and cross-correlation analysis, provides crucial information on the location, size, structure, and dynamics of the emitting regions, and shed light on physical mechanisms of particle acceleration and radiation emission.

In this section a quantitative analysis of the optical variability observed in PKS $0735+178$ is performed using 7 different methods in 13 different light curves. The aim of this work is to examine in detail the optical behavior on long-term timescales (months/years), using the whole 1906-2004 historical light curve, the pre-1970, portion and the best sampled 1970-2004 part, and to explore mid-term scales (days/weeks in intervals <200 days) using our 10-year monitoring data set with improved sampling. Each seasonal light curve in the best sampled $R$-band is analyzed separately (a part of the first 2 pilot seasons, where we obtained only few observations). For the first time a rather comprehensive temporal analysis of the optical variability was performed over 3 decades in time in this peculiar blazar, investigating scales between about 2 and 30 years regarding the historical dataset, and between a few days and about 100 days in the seasonal monitoring (the duration of a season with data point spans between 144 and 203 days). The following methods, optimized or adapted for unevenly sampled time series, are applied: the first-order structure function (SF), the discrete auto correlation function (DACF), the Lomb-Scargle periodogram (LSP), the discrete fourier transform in the "clean" implementation (CDFT), the phase dispersion minimization (PDM), the scalogram of the Continuous Wavelet Transform (CWT) using different waveforms, and the periodogram of the synthetic light curve constructed on the empty gaps, using different window functions (gaps window function periodogram GWFP).

The SF is equivalent to the power spectral density function (PSD) of the signal calculated in the time domain instead of frequency space, which makes it less dependent on sampling problems, like windowing and alias (see, e.g., Rutman 1978; Simonetti et al. 1985; Smith et al. 1993). The first order SF represents a measure of the mean squared of the flux differences $\left(F_{i}-F_{i+\Delta t}\right)$ of $N$ pairs with the same time separation $\Delta t$ :

$\mathrm{SF}^{(1)}(\Delta t)=\frac{1}{N} \sum_{i=1}^{N}\left(F_{i}-F_{i+\Delta t}\right)^{2}$

The general definition involves an ensemble average. Deep drops in the SF shape means a small variance and provides the signature of possible characteristic time scales, but a wiggling pattern and fake breaks (that can indicate false time scales) are common when the sampling is not sufficient. Typically, the SF increases with $\Delta t$ in a log-log representation, showing an intermediate steep curve, whose slope $b$ is related to the power-law index $a$ of the PSD by the relation $a=1+b$ (a typical PSD indeed has a power-law dependence $P(f) \propto 1 / f^{a}$ on the signal frequency $f=1 / t)$. The maximum correlation timescale is reached when the SF is constant for longer lags (Hughes et al. 1992; Lainela \& Valtaoja 1993).

The DACF allows us to study the level of auto-correlation in unevenly sampled data sets (see, e.g., Edelson \& Krolik 1988; Hufnagel \& Bregman 1992) without any interpolation or addition of artificial data points. The pairs $\left(F_{i}, F_{j}\right)$ of a discrete dataset are first combined in unbinned discrete correlations

$\mathrm{UDACF}_{i j}=\frac{\left(F_{i}-\langle F\rangle\right)\left(F_{j}-\langle F\rangle\right)}{\sigma_{F} \sigma_{F}}$,

where $\langle F\rangle$ is the average values of the sample and $\sigma_{F}$, the standard deviation. Each of these correlations is associated with the pairwise lag $\Delta t_{i j}=t_{j}-t_{i}$ and every value represents information about real points. The DACF is obtained by binning the 

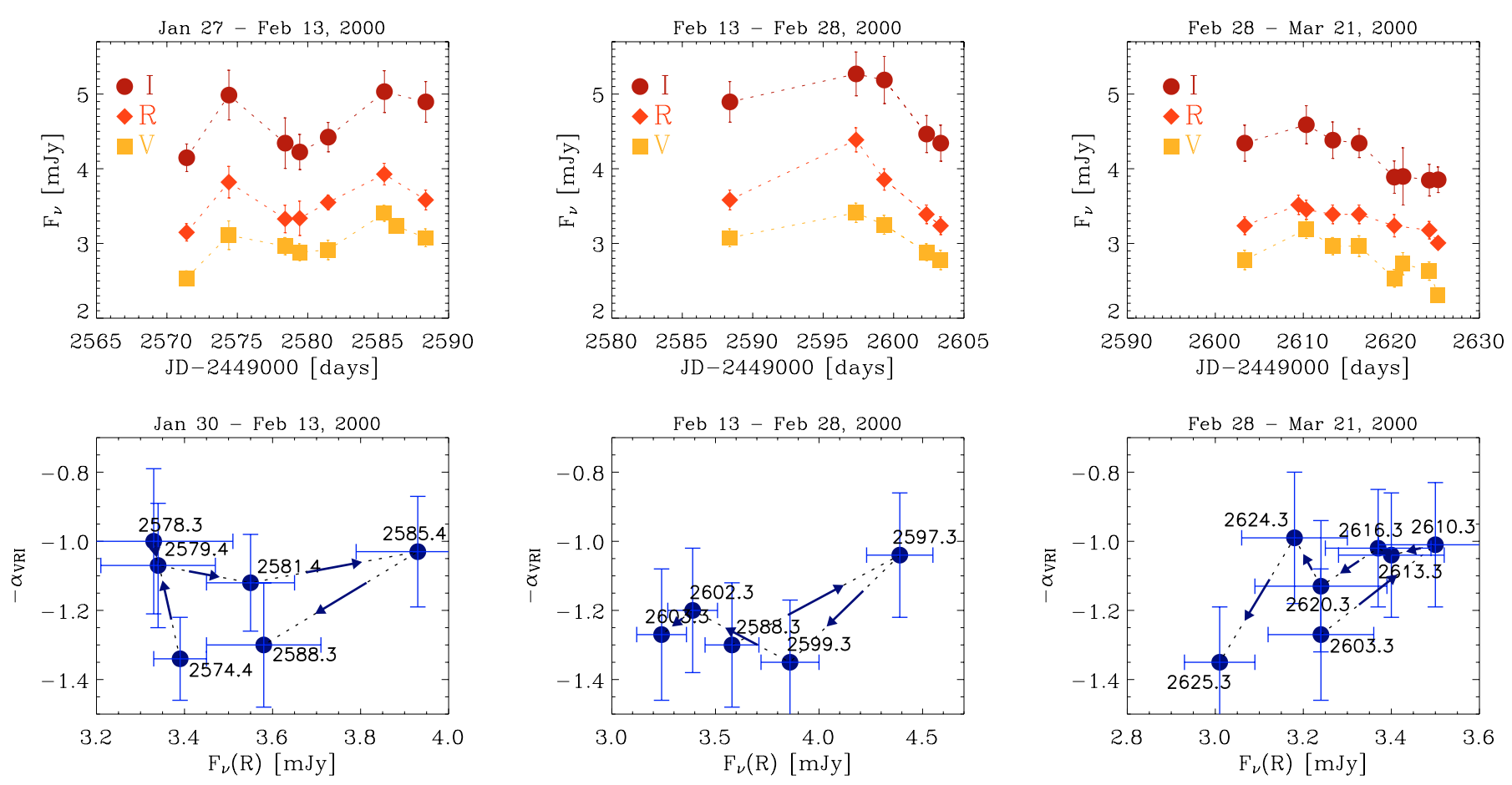

Fig. 6. Evolution of the continuum optical spectrum of PKS $0735+178$ (spectral index $\alpha$ ) as a function of the flux in the $R$-band, during 3 contiguous periods of flickering variability with moderate flaring activity observed by the same telescope (Perugia Observatory, in the period: January 29March 21, 2000). An approximative loop-like behavior of $\alpha$ is hinted in the first (clockwise) and in the third (counterclockwise) patterns.

$\mathrm{UDACF}_{i j}$ for each time lag $\Delta t$, and averaging over the number $M$ of pairs whose time lag $\Delta t_{i j}$ is inside $\Delta t$, i.e.: $\operatorname{DACF}(\Delta t)=$ $1 / M \sum_{i j} \mathrm{UDACF}_{i j}$. The choice of the bin size is governed by a trade-off between the desired accuracy in the mean calculation and the desired resolution in the description of the correlation curve. A preliminary time binning of data usually leads to better results. The number of the real points per time bin can vary greatly in the DACF, but data bins with an equal population can be built together with Montecarlo estimations for peaks and uncertainties, as done in the Fisher $z$-transformed DACF method (ZDACF, Alexander 1997).

The LSP is a technique analogous to the Fourier analysis for discrete unevenly sampled data trains, useful for detecting the strength of harmonic components with a certain angular frequency $\omega=2 \pi f$ (see, e.g., Lomb 1976; Scargle 1982; Horne \& Baliunas 1986; Papadakis \& Lawrence 1993). In the CDFT method, first a "dirty" discrete Fourier transform (DFT) for unequally spaced data is calculated and then an interactive "cleaning" of the dirty DFT is performed (see, e.g., Högbom 1974; Roberts et al. 1987; Foster 1995). The CDFT method is a complex and one-dimensional version of a deconvolution algorithm widely used in 2-dimensional image reconstruction. This technique provides a simple way to understand and remove false peak artifacts introduced by empty gaps. This method is effective especially in describing and recognizing multiperiodic signals. A standard (uncleaned) DFT method was implemented previously by Deeming (1975).

The PDM method (see, e.g., Lafler \& Kinman 1965; Jurkevich 1971; Stellingwerf 1978) tries to minimize the variance of data at a constant phase with respect to the mean value of the light curve. If a trial period is close to a real period, the scattering of data against the derived mean in the light curve constructed on such phase (the light curve folded on such a period) is small. The PDM method has no preference for a particular periodical shape; it incorporates all the data directly into the test statistic and it is well suited for small and randomly spaced samples. A value is statistically significant when the PDM drops towards zero.

The Wavelet method is used to transform a signal into another representation able to show the information in a more useful shape (see, e.g., Daubechies 1992; Foster 1996; Percival \& Walden 2002). Wavelet transforms (WT) permit a local decomposition of the scaling behavior in time for each quantity (in contrast to the usual methods based on the Fourier analysis), allowing the signal features and the frequency of their "scales" to be determined simultaneously. Hence it is a an especially useful tool to detect typical timescales and identify signals with exotic spectral features, transient information content, and nonstationary properties. WT are defined following the Fourier theory, but wavelets can be formally described as localized, oscillatory functions whose properties are more attractive than sine and cosine functions.

WT is computed at different times in the signal, using mother wavelets (orthogonal base functions localized in both time and pulse spaces) of different frequency and convolved on each occasion. In this way the power spectrum (i.e., the modulus of the transform value) on a two dimensional location-frequency plane is obtained (the so-called wavelet "scalogram"). A continuous WT of a one-dimensional (1D) time-series is computed as a complex array at different times, the real component being the amplitude and the imaginary component providing the phase. The square of the transformed modulus gives the wavelet power spectrum in function of both time and harmonic frequencies. In our analysis we chose the Morlet complex-valued waveform (see, e.g., Farge 1992), composed of a plane wave modulated by a Gaussian envelope of unit width:

$\psi_{0}(\tau)=\frac{1}{\sqrt[4]{\pi}} \mathrm{e}^{\mathrm{i} \omega_{0} \tau} \mathrm{e}^{-\tau^{2} / 2}$ 

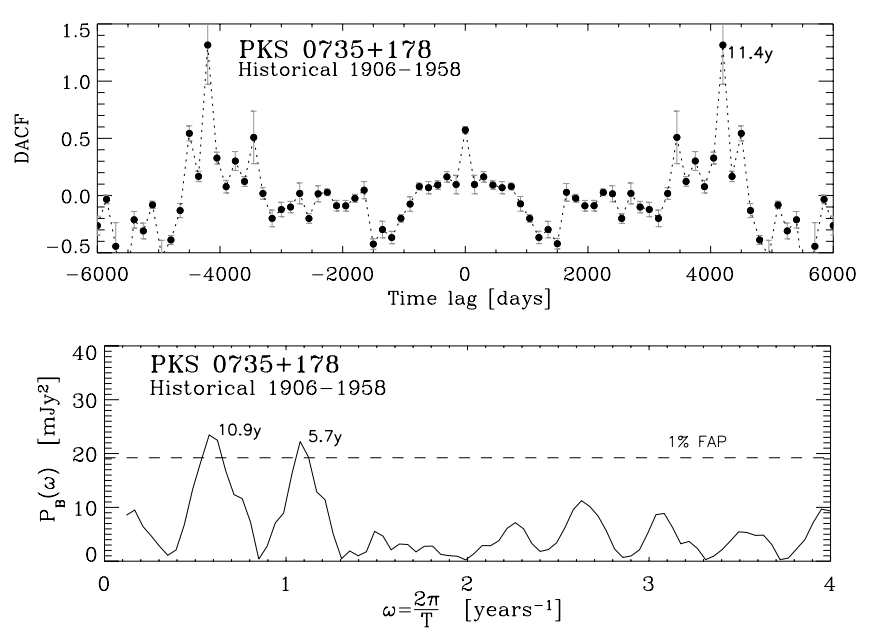

Fig. 7. Discrete autocorrelation function (DACF; data bin: 7 days, DACF bin: 150 days) and Lomb-Scargle periodogram (LSP; dashed line indicates the threshold of false alarm probability [FAP] fixed at 1\%) of the pre-1970 light curve. A characteristic timescale around 11 years is also hinted by this poorly sampled portion of the historical light curve.

where $\tau$ is the non-dimensional time parameter and $\omega_{0}$ the nondimensional frequency. Such continuous WT is convoluted on the discrete sequence of the time series $\left\{F_{i}\right\}$, with scaled and translated versions of $\psi_{0}(\tau)$. It is considerably faster to calculate such continuous WT in Fourier space: the convolution theorem allows us to do all the $N$ convolutions for a given scale simultaneously and efficiently in Fourier space, $N$ being the number of points in the time series) using a standard DFT (Kaiser 1994; Percival \& Walden 2002).

The temporal analysis of the PKS $0735+178$ optical dataset is performed using all the methods mentioned above separately on each light curve with results summarized in Table 3 . In particular the diagrams from the time-series analysis of 5 light curves (the whole historical 1906-2004 and best sampled 1970$2004 B$-band series, and the seasonal IV, VII and X $R$-band light curves from our dataset) are reported in Figs. 8-12. In the analysis of mid-term scales, the problem of spurious artifacts given by seasonal gaps with no data (solar conjunction with the source) was avoided, studying each seasonal light curve separately. The GWFP of the best sampled 1970-2004 light curve (Fig. 9 last panel, bottom, right) shows indeed only one powerful peak placed at exactly 1 -year scale. This represents the periodical yearly recurrence of the seasonal gaps.

The whole 1906-2004 light curve (Fig. 8) is patently affected by substantial differences in data sampling, by void gaps, by a poor sampling earlier than 1970, and by long empty interval (1958-1970). Nevertheless, several signal features and characteristic timescales are pointed out by the different techniques (using both binned and unbinned series): about 8.6y, 12-13y, $25 \mathrm{y}$, and $34 \mathrm{y}$ (see the summary reported in Table 3 ). The $13.7 \mathrm{y}$ timescale (pointed out by nice features in the LSP and CWT functions) is the same value claimed as the major component of a multi-periodical trend by Qian \& Tao (2004) and Fan et al. (1997), on the other hand the $8.6 y$ timescale (suggested by the DACF and LSP) is probably to be ascribed mainly by the temporal behavior after 1970 (Fig. 9), and it is reported by Qian \& Tao (2004), too. Longer duration scales (e.g., 34 y) are difficult to be set out with confidence. Fake signal features, due to recurrences and temporal patterns given by the empty gaps, occurred at scales shorter than 8 years only (see the GWFP plot); therefore minor and fainter hallmarks in the SF, LSP, CWT corresponding to such shorter scales are neglected.

The best sampled portion of the historical light curve (19702004, Fig. 9) spans 33 years and has a quite fair continuous, regular, and long-term coverage: 867 nights with 1 datapoint at least, an average number of data points per night of 1.8 , an average gaps among data of 7.8 days, and a maximum gap of 1.6 years (Table 3 ). The more relevant characteristic timescales suggested by the different temporal analysis methods are around 4.5, 8.6, and 12.5 years (an other few values such as 3.5, 7.4, and 11.8 years could be traced back to the previous mentioned, if we consider the finite-resolution accuracy of these methods). The 4.5, 8.6, 12.5 years scales might be considered multiple harmonic signatures of a fundamental (coherent, absolute, or transient periodical, or again with drifting duration) component of about 4 years (see also the spline visual envelope in Fig. 13). A characteristic timescales of about 4.8 years was also previously claimed by Webb et al. (1988) and Smith et al. (1987), while the 8.6 year scale was recently suggested by Qian \& Tao (2004). On the other hand no evidence for the periodical signature of 14.2 years previously claimed by Fan et al. (1997) are observed, but only weak hints for scales in the 11.6-13.5 years range are found out. The power spectral density in the $1 / f^{a}$ regime shows a slope index $a$ between 1.5 and 2 (i.e., next to pure shot-noise behavior). The GWFP show a very powerful fake signature at 1.0 years as expected, produced by the recurrent 1-year gap between subsequent observing seasons. This artifact due to sampling it is not completely neglected by the methods used (see, e.g., the residual peaks around $\omega \sim 6$, i.e., $\sim 2 \pi$ in the LSP plot), therefore it is very useful to develop and make use of the GWFP technique in conjunction with the other time series methods. No other (longer) fake features due to the irregular sampling are pointed out by the GWFP, therefore all the other characteristic timescales claimed in this light curve can be considered due to real variability. In the CWT scalogram a significant and localized pulse in power is visible (with a scale around 4.8 years and located in correspondence of the big 1977 outburst). Another localized bump gives a scale of about $7.4 \mathrm{y}$. An elongated and lower intensity band in the CWT scalogram, corresponding to the last epochs (about 19942004) again indicates a coherent time scale between the values 8.2-8.9 years. Results are quite similar using different suitable CWT mother functions as the Mexican-hat and Paul waveforms in this light curve.

As example, in the time series analysis of the IV observing season of our $R$-band dataset (Sept. 1995, Apr. 1996, Fig. 10) a main modulating trend quite monotonically decreasing is observed. This is stated for example by the shape of the SF in the logarithmic plot (second panel, top right of Fig. 10) being quite linearly monotonic and without any turnover flattening (given by the reaching of a maximum correlation timescale). In this general trend 4 or 5 moderate and secondary oscillations can be visually identified (possibly related to the unique and weak signal of a characteristic timescale between 28-34 days, as hinted by the DACF, PDM, and CWT). Other characteristic scales are not displayed, and the artifact noise given by the irregular gaps is important only at timescales below 13 days (see the GWFP last panel, bottom right). The power spectral density in the $1 / f^{a}$ regime, shows a power index $a=1.97 \pm 0.25$, i.e., a temporal variability mode like the shot noise (brown noise) signal.

In the $R$-band light curve during the VII observing season (Oct. 1998, May 1999, Fig. 11) a brightening stage (between about JD $=2451170$ and JD $=2451265$, i.e., 95 days long) seeming to be produced by two blended main flares of about 50 days duration, might be supposed by a visual inference. 

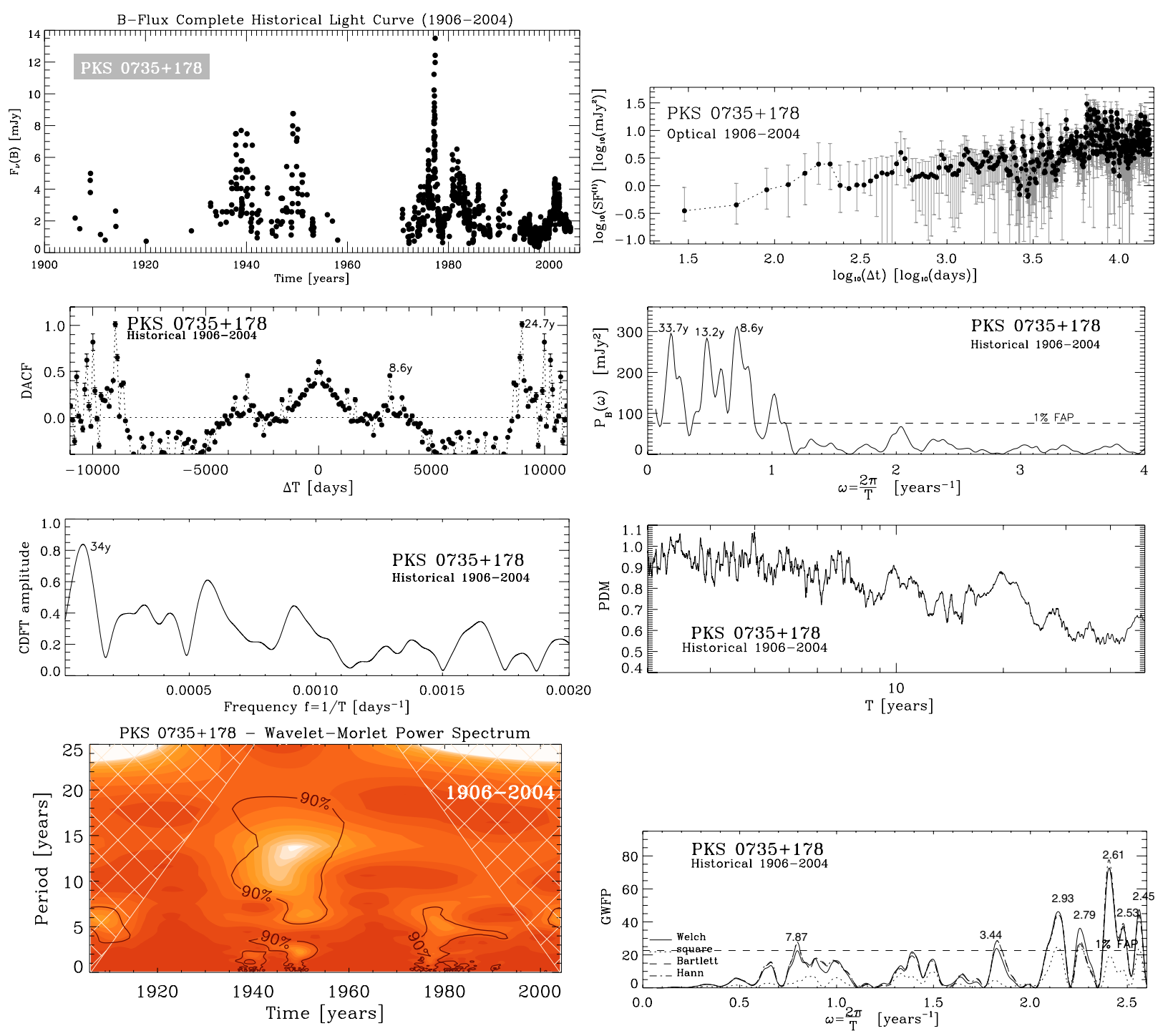

Fig. 8. Panels from left to right and following below: the whole historical (1906-2004) flux light curve of PKS $0735+178$ in the $B$-band and plots from the related time-series analysis. First order structure function (SF; data bin: 7 day, SF bin: 60 days) in log-log representation, discrete auto correlation function (DACF; data bin: 7 day, DACF bin: 90 days), Lomb-Scargle periodogram (LSP; dashed line is the 1\% false alarm probability [FAP] threshold), "clean" implementation of the discrete fourier transform (CDFT), phase dispersion minimization function (PDM), plane contour plot of the wavelet scalogram (i.e. the two-dimensional energy density function $|C W T(t, T)|^{2}$, CWT being the continuous wavelet transform computed using a Morlet waveform, $t$ and $T$ the time and period scale, respectively). In the last panel the periodogram of the synthetic light curve constructed upon the empty gaps using different window functions (GWFP; gap threshold 1 year). In the CWT scalogram the power spectral density is represented by filled-color contour levels, while the thick black contours are the $90 \%$ confidence levels of true signal features against white/red noise background, and the cross-hatched regions represent the "cone of influence", where edge effects become important. The descriptions of the plots above are applied to the following Figs. 9-12.

Such values (about 95 and 50 days) are pointed out by the SF, DACF, LSP, PDM, and CWT functions (Table 3) as characteristic timescales (scales where more power is in the signal). The SF and PDM methods also suggests a possible timescale of about 30 days. Fake features given by the irregular gaps are important only at timescales below 21 days (GWFP last panel, bottom right of Fig. 11). The power spectral density function shows a power index $a=1.64 \pm 0.09$, i.e., a temporal fluctuation mode placed halfway between the flickering and the shot noise behavior.

The plots from the analysis of the $\mathrm{X}$ observing season data (Oct. 2001, May 2002, Fig. 12) obtained by our monitoring program, show a high state with two relevant flares, followed by a low and rather oscillating phase (after date JD $=2452273$ ). Unfortunately there was a long observing gap (about 25 days between JD $=2452218$ and JD $=2452243$ ) during the more active phase of this season, which is the brightest optical state of the source recorded in our 1994-2004 database, too. Characteristic timescales of about $52-55$ days are suggested by the LSP, CDFT, and PDM, while a 41 day scale is also found by the DACF and LSP (Table 3). Fake features given by the irregular gaps are influential only at timescales below 18 days (GWFP last panel, bottom right, Fig. 12). The power spectral density function shows a power index $a=1.46 \pm 0.17$, i.e., again a temporal fluctuation mode placed halfway between the flickering and the shot noise behavior.

The summary of the temporal statistics and analysis results is shown in Table 3. The following information is reported about statistics: the observing interval and its duration (98.1 years in total, and between 144 days and 203 days regarding our observing seasons); the number of the effective observing nights with 

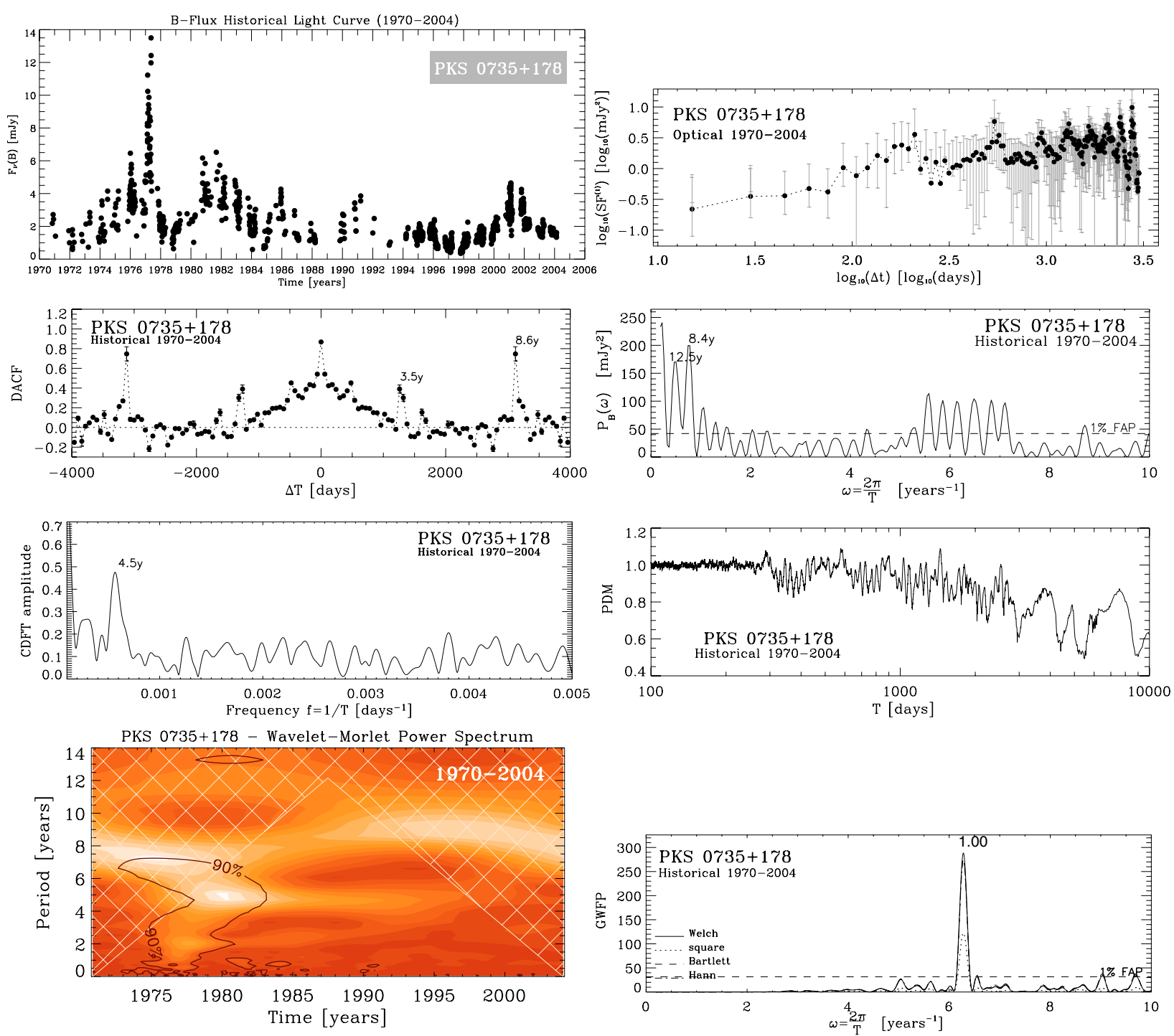

Fig. 9. Panels from left to right and following below: the whole historical (1970-2004) flux light curve of PKS $0735+178$ in the $B$-band and plots from the related time-series analysis. SF (data bin: 1 day, SF bin: 15 days), DACF (data bin: 1 day, DACF bin: 60 days), LSP, CDFT, PDM, Morlet-CWT scalogram, and GWFP (gap threshold 0.3 years).

one data point at least (867 data points in the 1970-2004 light curve, and between 20 and 62 in our dataset observing seasons); the average number of data points per observing night in the interval (spanning between 1 and 2.3, implying no data clustering); the average separation between 2 successive data points ( 3 days on average on all our 10 observing seasons); the maximum separation (maximum empty gap) between 2 successive data points (no more than 25 days in the worst sampled season). In time intervals where the SF slope can be recognized in the log-log representation, we calculated its power index $b$ trough a linear regression. The following quantities are reported (when possible) about the time series analysis: characteristic timescales calculated by deep drops in the SF, the power-law index $a=1+b$ of the PSD in the $1 / f^{a}$ regime calculated by the SF, characteristic timescales inferred from the SF turnover to the plateau produced by times longer than the maximum correlation lag, timescales estimated from power peaks in the DACF/ZDACF, in the LSP, and in the CDFT, and timescales indicated by deep drops in the PDM and by peaks in the CWT scalogram.

The criteria adopted to possibly avoid quoting fake features and artifacts due to the irregular sampling and gaps, or edge effects, in Table 3 are the following: 1) only timescales shorter than $1 / 2$ or $1 / 3$ of the interval duration were considered; 2 ) only the more relevant signatures in each method are preliminary considered; 3 ) among these we discarded the features that are not indicated by more than one method on the same light curve portion, when the signature is not particularly strong; 4) if some timescales, among the remaining, are still matched by the synthetic GWFP, these are discarded, too. Most of the applied methods take the power of artifacts given by the irregular dataset and recurrences of gaps well into account, as shown by Figs. 8-12 (see the comparison of the first 7 panels with the last GWFP panel). However residual spurious power can still be present in the functions (see, e.g., the spurious peaks of the LSP around $\omega \sim 6.3$, i.e., $T=1$ year, in Fig. 9 as mentioned).

On long timescales the main temporal components we found can be grouped in three main ranges of values: between 4.4 and 4.8 years, between 8.2 and 8.6 years, and between 10.8 and 13.2 years (Figs. 7-9 and Table 3). These components could modulate the long-term optical light curve of PKS $0735+178$ with roughly cyclical oscillations. A characteristic timescale of about 4.8 years was previously claimed as well by 

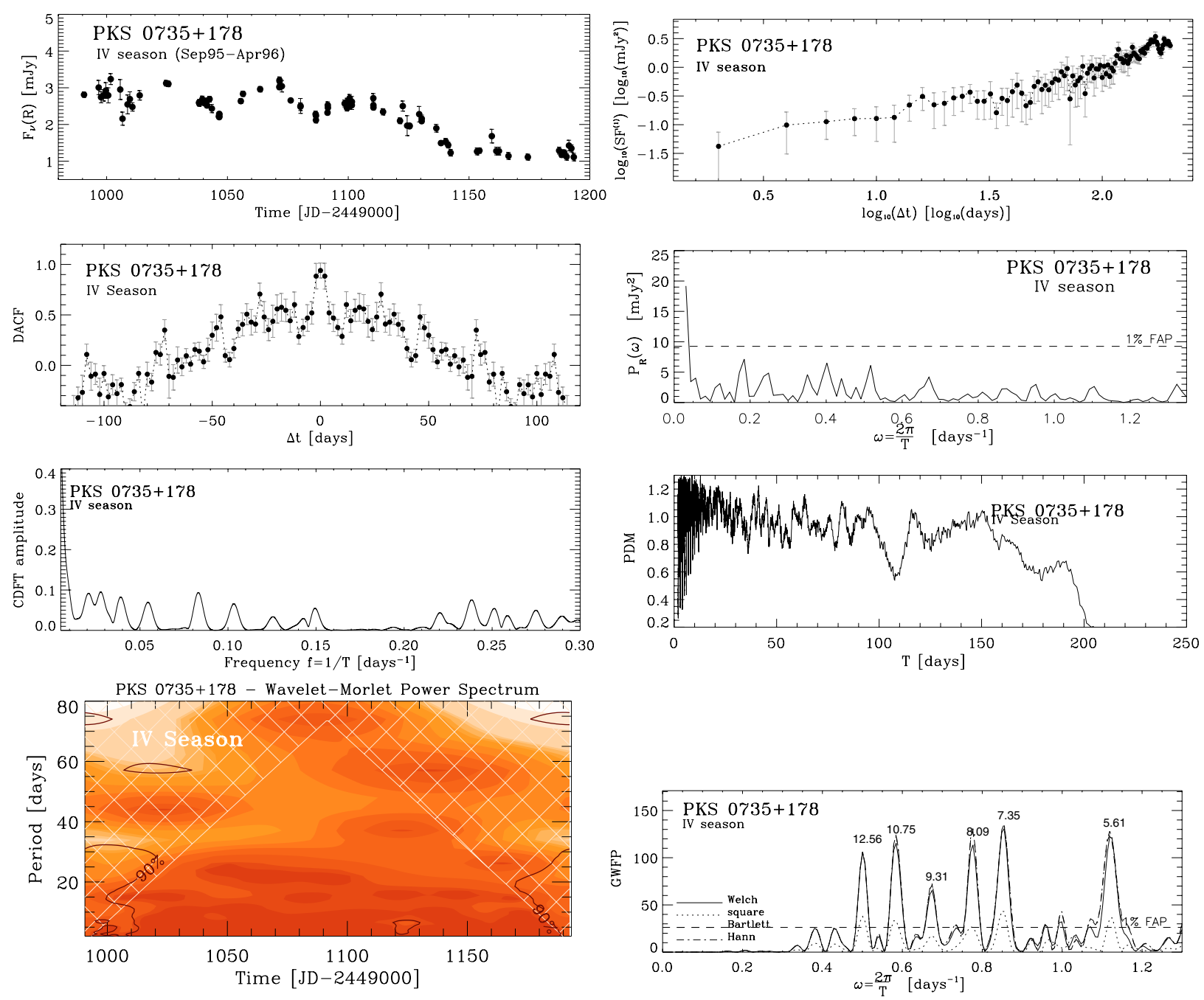

Fig. 10. Panels from left to right and following below: the $R$-band light curve of PKS 0735+178 in the IV observing season (Sept. 1995, Apr. 1996) obtained by our monitoring program, and related functions produced by the time-series analysis. SF (data bin: 1 day, SF bin: 2 days), DACF (data bin: 1 day, DACF bin: 2 days), LSP, CDFT, PDM, Morlet-CWT scalogram, and GWFP (gap threshold 5 days).

Webb et al. (1988) and Smith \& Nair (1995) while a 8.6-year scale was recently suggested by Qian \& Tao (2004). Then again we did not find any strong evidence of further and longerduration ( $>14$ years) timescales reported in literature (e.g., Fan et al. 1997; Qian \& Tao 2004, and Sect. 2), but only weak hints of a 25-year and a 34-year signature.

Some parts of the light curve could contribute with different typical scales to the overall series, while data are treated in varying ways, with timescales suppressed or enhanced as the relative weight of different segments of the light curve change. The variability scale of $8.2-8.6$ years could be an important finding and a real signature of a dominant and possibly quasi-periodical component anyway, because it was found in both the whole 19062004 series and its best-sampled portion. Moreover some uncertainty and statistical dispersion in the values found by different methods is expected, especially when the sampling is irregular, and here the dispersion range is small ( 0.4 years). In addition, fast and/or isolated flares randomly occurred and are uncorrelated to any general trend, can provide loud contributions to the power spectrum, disproving any periodicity hypothesis based on a long but under-sampled historical light curve. The better sampled portion of the PKS 0735+178 light curve did not disprove the characteristic timescale mentioned above, therefore it is reasonable to suppose a possible dominant pseudo-period of around 8.5 years. This hypotheses is open to future investigations based on prolonged monitoring observations. In this view the shorter 4.4-4.8-year scale found might be a submultiple of the previous component. Hence this would be the real period of the fundamental harmonics (pointed out only by the best sampled 1970-2004 portion because of the sufficient sampling to detect it). A corroboration of this conjecture is also provided by the spline interpolation on the light curve reported in Fig. 13: 6 major maxima and cycles are outlined between 1970 and 2004. Using a 600-days binning and considering the separation between the troughs of this pseudo-sinusoidal envelope, we have cycles of about 6.0-6.5 years and 3.9-4.8 years. This raw visual interpretation could be linked to the possible fundamental (and duration-drifting) modulating component of 4.4-4.8 years found with the detailed time series analysis. Finally the group of longer-duration scales found in the broader range 10.8-13.2 years are detected in each piece of the historical light curve (see Table 3), but these values are probably a bit much scattered to mask a strict periodicity signature.

On intervals shorter than 200 days, monitored by our observations in 10 subsequent seasons (from the III starting in Oct. 1994 to the XII ending in Feb. 2004) and analyzed deeply 

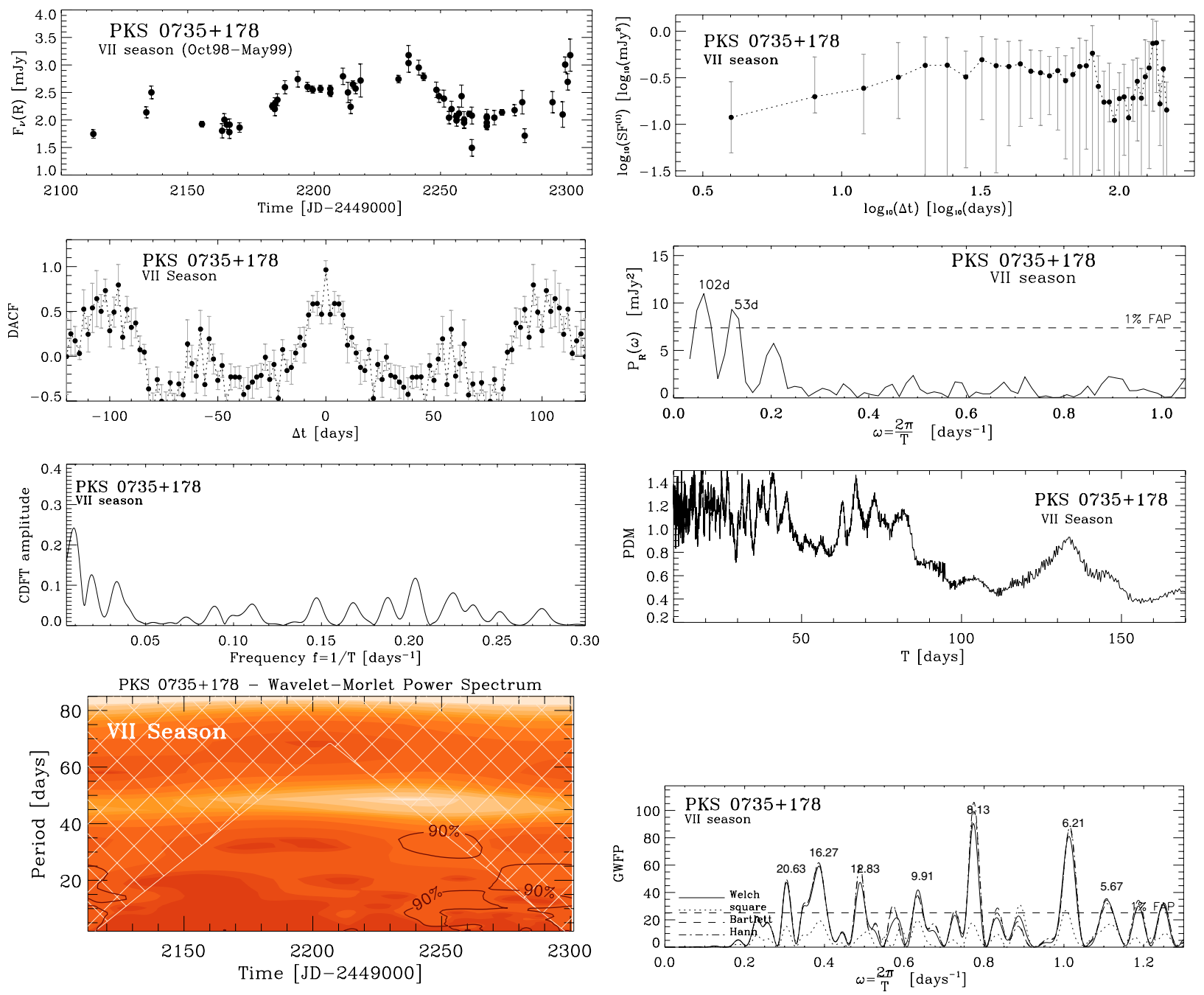

Fig. 11. Panels from left to right and following below: the $R$-band light curve of PKS 0735+178 in the VII observing season (Oct. 1998, May 1999) obtained by our monitoring program, and related functions produced by the time-series analysis. SF (data bin: 1 day, SF bin: 4 days), DACF (data bin: 1 day, DACF bin: 2 days), LSP, CDFT, PDM, Morlet-CWT scalogram, and GWFP (gap threshold 5 days). Issues and results from these diagrams are described in the text.

with the methods mentioned above, there is no evidence for one single and pure periodical feature, but there are signatures of several characteristic timescales of mid duration, commonly found in different observing seasons. These "recurrent" and "common" timescales are distributed among few groups of values: 18 days, 24-25 days, 27-28 days, between 40 and 42 days, between 50 and 56 days, 65-66 days, between 76 and 79 days, and 95-96 days. In particular the timescales of 27-28 days are found in 3 observing seasons (might be related to the synodical month interference), timescales between 50 and 56 days are observed in 6 seasonal light curves, and timescales between 76 and 79 days are detected in 4 seasons. Several hypotheses can be proposed, about these timescales. 1) These temporal signatures could be the result of rough multi-periodical behavior given by the superimposition of few harmonic components (spanning from about 2 dozen days to about 100 days). 2) They could be produced by pseudo-periodical cycles, with a drift of the period duration around a fundamental value of 27 days for example (50-56 and 76-79 days could be thought of as rough multiples in this case). 3) Such characteristic timescales could be produced by different periodical stages of transitory nature, with a sort of time-localized periodicity surviving only for limited epochs.
4) Again they could be the result of a variability mode endowed with a few and typical duty-cycles, showing similar/recurrent peak shapes/durations, and occurring at random times and partly mixed in the light curve. This type of variability is characterized by an overall PSD slope placed between the flickering and the shot noise, and could be related to turbulence mechanisms and to a succession/superimposition of optical emitting blobs providing a quite nonhomogeneous and lumpy/knotty structure of the jet at the optical bands. In this interpretation the exceptional big flares/outbursts observed in optical light curves of blazars, are considered as simple statistical tails of the same process. This is opposite to theories that foresee big and rapid flares produced by mechanisms of different nature, with respect to the processes responsible for the common mid-flaring/flickering activity, but yielding the same "red/brown" shape of the PSD, when combined. As cited above the sampling of our optical data allowed to investigate timescales between about 1 week and about 100 days, and the characteristic timescales we found in Table 3 roughly span in the range $t_{\mathrm{var}}=20-90$ days. This means typical sizes of the optical emitting regions $R \sim c t_{\mathrm{var}} \mathcal{D} \sim 10^{17-18} \mathrm{~cm}$ (i.e., pc-scale jet, $\mathcal{D}$ being the bulk Doppler factor). 

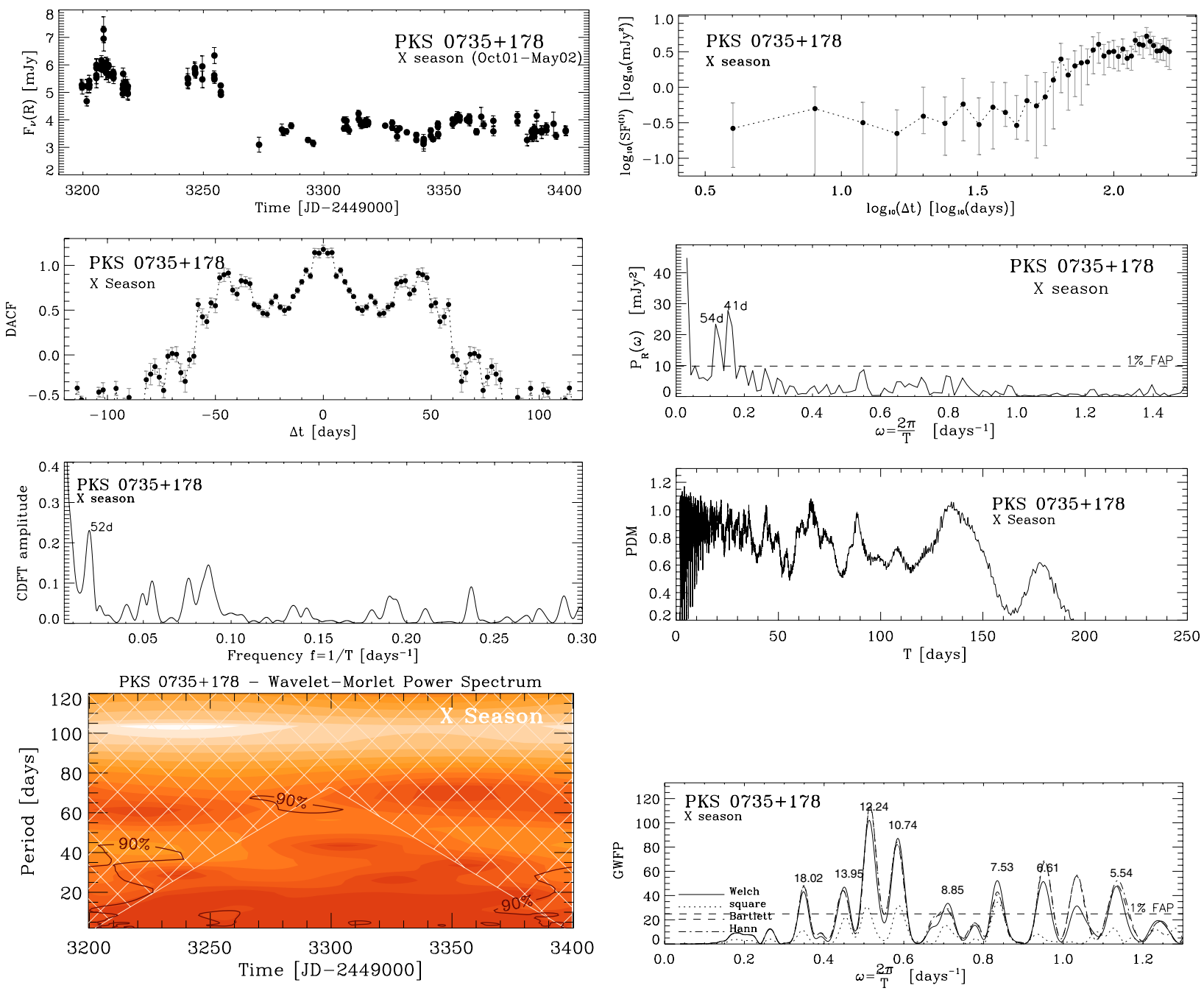

Fig. 12. Panels from left to right and following below: the $R$-band light curve of PKS $0735+178$ in the $X$ observing season (Oct. 2001, May 2002) obtained by our monitoring program, and related functions produced by the time-series analysis. SF (data bin: 1 day, SF bin: 4 days), DACF (data bin: 1 day, DACF bin: 2 days), LSP, CDFT, PDM, Morlet-CWT scalogram, and GWFP (gap threshold 5 days).

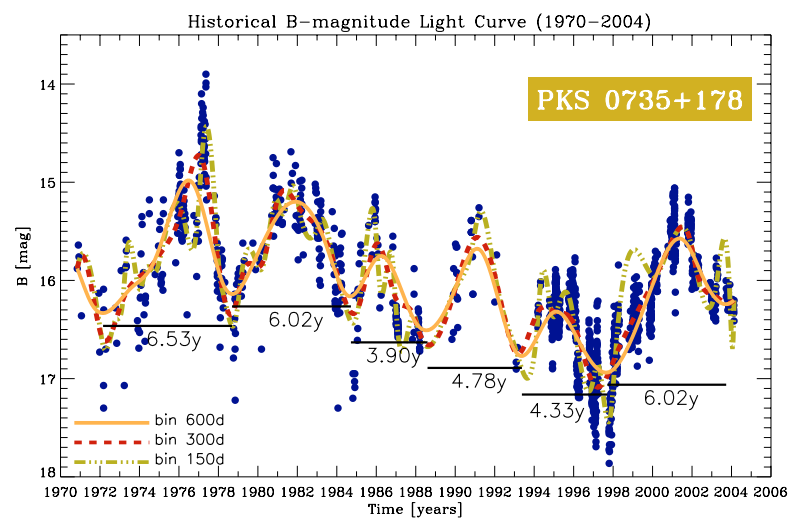

Fig. 13. Cubic spline interpolations of the best sampled 1970-2004 historical light curve of PKS $0735+178$. The continuous (orange) spline curve is obtained with a data binning of 600 days, while the other dotted/broken spline lines are obtained with binnings of 150 and 300 days. The interpolations show 6 main peaks already visible with the 600-days bin.

\section{Summary and conclusions}

Blazars are one of the most exciting class of AGN, and the primary known extragalactic sources emitting high energy gammarays. Variability monitoring is an important effort in the study of blazars for several reasons (even if well-sampled light curves are a very big challenge to be obtained at optical wavelengths normally). 1) Short-term observations and multiwavelength (MW) snapshots obtained during broad but limited-duration campaigns cannot resolve all the puzzling questions about blazars, while long-term monitoring allows us to investigate the behavior and evolution of the emitted flux on different scales. 2) The knowledge about time variability is crucial, like the spectral variability in constraint emission models, and the observed behavior on long scales can be cross-correlated with the MW observations usually available on short timescales. 3) Radio-optical monitoring could be considered a farsighted effort, too: it enables us to construct long-term records of variability for several sources, useful for future research. 4) Even if most of blazars seem to exhibit an irregular, uncorrelated, and unpredictable temporal behavior, their optical light curve shapes appear to be not trivial (sometimes signatures of long-term memory, temporal self 


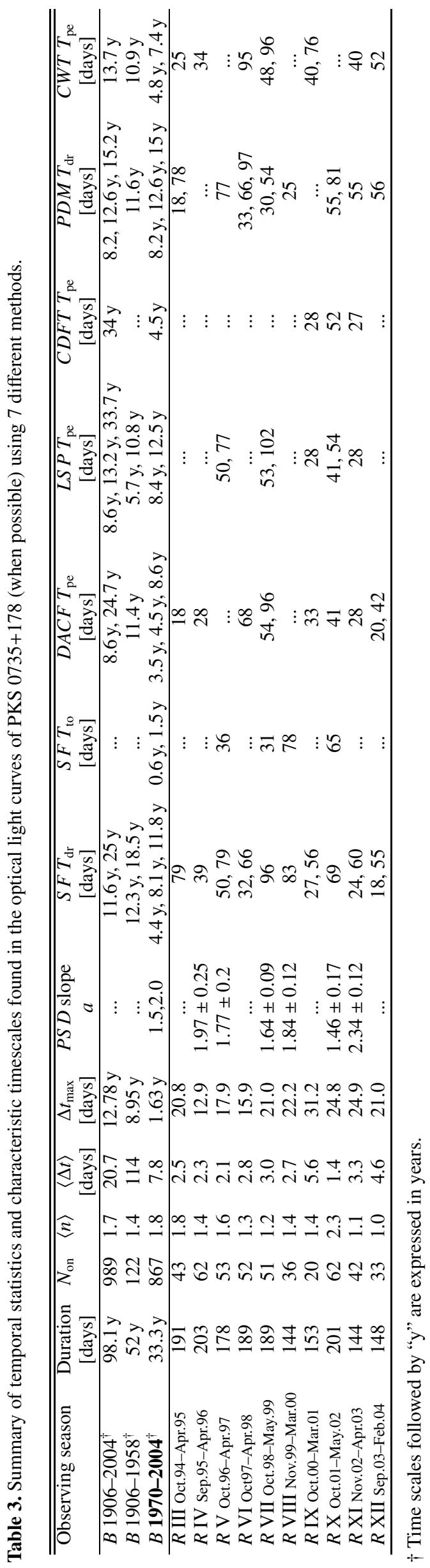

similarity, and intermittence are displayed), whereas in a few known cases periodical/quasi-periodical components cannot be ruled out. 5) In addition, flare triggers and target of opportunity alerts for space observatories and large-size telescopes are usually based on a regular and constant monitoring. 6) Time series analysis of sparse data sets (like blazar light curves), is a challenging, interdisciplinary subject, being developed and applied on a wide variety of present-day research topics outside astrophysics. 7) Our fairly novel investigation and results on midterm optical timescales (days, weeks), could be also compared to the analysis of blazar gamma-ray light curves that will be provided, at the same scales, by the forthcoming Gamma-ray Large Area Space Telescope (GLAST). In fact this high-energy space observatory will be a large field-of-view and all-sky monitor for flares and variability, allowing us to record flux variations over timescales $>1$ day on hundreds of $\gamma$-ray blazar-like sources. 8) Moreover worldwide international collaborations and the participation of amateur and schools'/universities' optical telescopes are now possible (thanks to the development of CCD photometry and automation technology) meaning a valuable link for education and public outreach.

With this in mind, during a long-term and painstaking optical monitoring program, we have obtained, collected, and analyzed the largest amount of optical data in 4 colors ever published on the prominent blazar PKS $0735+178$, thanks to the collaboration of 3 professional observatories (Perugia, Torino, and Tuorla) and 1 amateur facility (Sabadell). Furthermore, a new VRI photometric calibration of 7 comparison stars in the field of this blazar is presented (Table 1), joined with the reconstruction and analysis of the whole historical light curve (now spanning from 1906 to 2004). These optical data are rather unique with respect to continuity, sampling, and duration for this source, and the associated data analysis is comprehensive enough, despite natural difficulties (weather/seeing conditions, seasonal gaps, technical problems, or limited manpower). About 500 nights of observations, collected over more than 10 years (period 1993-2004), and providing 1332 new BVRI final data points on PKS 0735+178 are reported and investigated, aiming to provide a quantitative statistical description of the data set, a characterization of the multi-band behavior, and an investigation of variability over 3 decades in time, for the first time in this blazar.

During the last 10 years, PKS $0735+178$ continued to show rapid and large-amplitude optical variations typical of blazars, even if the source remained in a rather low or intermediate brightness state (mag $R>14$ ), showing a mild flaring activity. However starting from the end of 1997 the source showed a clear increase of the average brightness until 2001, when an active phase occurred. In this decennium typical variations of about 2 mag in less than half-year are observed joined with a general wiggling pattern produced by a superimposition or succession of flares, and modulated by a slower (possibly achromatic and oscillating) long-term trend. The quiescent and mild-activity reported from 1994 to the second half of 2000 in the optical band was also pointed out recently in radio bands, as a period of quiescent flux activity and highly twisted jet geometry (Agudo et al. 2006). In the whole $\sim 100$-years history of PKS $0735+178$ the five brightest outbursts and active phases were observed: the last occurred in the period Feb. 2001-Oct. 2001, and the brightest outburst was observed in May 1977 (when the source reached its historical optical maximum $B \simeq 13.9$ ).

The analysis of the continuum optical spectrum of this blazar suggests, as expected, a correlation between the fluxes in the $B$ - and $R$-bands, while the long-term variability of the spectral index $\alpha$ appears to be essentially achromatic and independent 
of the wavelength (Fig. 5). High-amplitude and isolated flares can imply correlated spectral changes (usually a flattening, i.e., bluer when brighter), but our data showed usually a rather erratic evolution of $\alpha$ as a function of the flux, and few or weak hints of non-thermal signatures (see, e.g., Fig. 6). At these mid-term timescales and without an increased sampling, it is reasonable to expect that the superimposition of pure synchrotron optical fluctuations and emission peaks cannot easily be disentangled from slower variability patterns produced by different mechanisms.

A summary of the quantitative temporal analysis performed in each single observing season of our $R$-band light curves is reported in Table 3. Summarizing data sets investigated are: 3 historical $B$-band flux light curves (the complete 1906-2004 curve, the best sampled 1970-2004 part, and the 1906-1958 portion), and 10 separated $R$-band flux light curves obtained in each observing season of our monitoring program (from the III season that started in Oct. 1994 to the XII season ended in Feb. 2004). The following data are reported in the columns of Table 3 (from left to right), for each single light curve: (1) optical band and name of the light curve interval; (2) duration of the light curve; (3) number of the effective observing nights $N_{\text {on }}$ with one data point at least; (4) average number of data points per observing night $\langle n\rangle$; (5) average separation between two successive data points; (6) maximum separation (maximum empty gap) between two successive data points; (7) power-law index $a$ of the power spectral density (PSD) in the $1 / f^{a}$ regime $(f=1 / T)$, calculated in the time-domain through the first order structure function $S F$; (8) characteristic timescales $T_{\mathrm{dr}}$ calculated by deep drops in the $S F$; (9) characteristic timescales $T_{\text {to }}$ inferred from the $S F$ turnover to a long-lag plateau in the log-log representation; (10) timescales $T_{\mathrm{pe}}$ estimated from peaks in the discrete auto-correlation function $(D A C F)$; (11) timescales $T_{\mathrm{pe}}$ derived by peaks in the Lomb-Scargle periodogram $L S P$; (12) timescales $T_{\text {pe }}$ derived by peaks in the "clean" implementation of the discrete Fourier transform $(C D F T)$; (13) timescales $T_{\mathrm{dr}}$ estimated from deep drops in the phase dispersion minimization function PDM; (14) timescales pointed out by peaks in the 2D contour plot of the wavelet scalogram (the two-dimensional energy density function provided by the continuous wavelet transform $C W T$ computed using a Morlet mother waveform). The errors in the timescales are difficult to estimate in general, and the significance of a scale is high when it is relevant according to a method, and when several methods point out similar values. Columns from (2) to (5) show that the sampling is fairly regular without high data clustering.

Intervals $<200$ days are investigated discovering quite common characteristic scales of variability, falling especially into value ranges of 27-28 days, 50-56 days, and 76-79 days. These signatures are the stronger contribution to the power spectrum, possibly produced by correlated flares with typical duty cycles emitted by some charge/discharge-like mechanisms. On the contrary, these typical timescales might represent the effects of a single or few powerful random events that are completely uncorrelated and infrequent. In other words, even if a characteristic and intrinsic timescale is found, this does not necessarily mean a discovery of a dominant modulation or periodicity. In fact such evidence could well be produced by events of a random or transitory nature, or be misrepresented by a combination of different underlying components affected by an insufficient observing sampling.

Moreover the shot-noise (Brownian/brown noise) behavior, pointed out by the values of the power-law index $a$ of the PSD (computed in each season and falling between 1.46 and 2.34, see Table 3), reflects the nature of the variations and can be linked to the findings cited above. Red (flickering) and brown noise are termed usually as $1 / f^{a}$ (power-law decline) fluctuations, meaning that the occurrence of a specific variation is inversely proportional to its strength. Brownian variability can be produced by a sequence of random pulses endowed with long-term memory, where independent/discrete events produced in different spatial sites, and parallel relaxation processes (like shocks and emitting knots, electron density fluctuations, magnetic field turbulence or plasma instabilities) might generate a succession of mild optical flares and oscillations with similar duty-cycles, on the mid-term timescales we investigated. In this view, isolated, big and rapid flares/outbursts can be produced by the same mechanism responsible for the mild-flaring (flickering/shot-noise, i.e., turbulencelike) variability, being interpreted as tails of the statistics, rather than events of completely different nature. We remark that fluctuation in the statistical moments and parameters like the PSD and variance may be intrinsic in red/brown noise processes (see, e.g., Vio et al. 2005), even if our analysis is essentially phenomenological and model independent. In addition when we analyzed different segments of the light curve separately and applied methods like the wavelets, we took partly into account non-stationarity problems.

In reference to the historical behavior of PKS 0735+178, we found 3 main characteristic timescales having an extension of about 4.5-year, about 8.5 years (possibly signatures of the same 4.5 years fundamental component), and between about 11 and 13 years (Table 3 ). These scales could be the result of an oscillating and achromatic trend, modulating (with a pseudo-periodic or multi-component course) the long-term variability. Pseudoperiodicity can imply drifts in duration and modulation, while a multi-component trend has several different scales contributing in the composite modulation. In particular in the first hypothesis, the majority of the long-term characteristic timescales found might be multiple signatures of a base component slightly drifting and varying around the value of 4.5 years. The visual inference based for example on the light curves of Figs. 4 and 13, can support this statistical finding. A rather "humped" or "multi-bumped" cyclical activity is evident, possibly meaning a bimodal course, defined by an alternation of active and quiescent stages. The limited temporal range of observations having a sufficient sampling (1970-2004) does not permit us to understand if this pseudo-cyclical activity is a stable or transitory phenomenon, and several hypotheses can explain the observed trend. A quasi/pseudo-periodical behavior (with a fundamental component drifting/oscillating around a value of 4.5 years); a multi-component modulation (by possibly different correlated mechanisms); a mere random or transitory occurrence; a combination of the previous scenarios (for example a mixture of a multi-component trend with a quasi-periodical component). The achromatic behavior reported in Sect. 7, is in agreement with a dynamical model implying slower variations of the base level flux and long-term modulations, resulting in variations in the beaming factor of the jet.

This kind of optical course could be better correlated to the radio flux behavior and the twisted (maybe precessing) jet of PKS $0735+178$ observed in detail from several years. This peculiar blazar (both radio and X-ray selected, and also a gammaray EGRET source) shows quite slow variations in the radio bands and a complex morphology displaying several moving components. In previous literature the optical and radio history of PKS $0735+178$ have already suggested a possible periodical activity. Periodicity in blazars has been debated for more than 40 years and several models were developed to explain this prospect (for example precessing or helical jets and 
supermassive binary black holes, see, e.g., Lehto \& Valtonen 1996; Sillanpää et al. 1996; Villata et al. 1998; Rieger \& Mannheim 2000; Valtaoja et al. 2000; Ostorero et al. 2004). However, only in very few (and well publicized) cases still there is a sufficient evidence of cyclical outburst (like in OJ 287), and usually there has been usually a general scepticism about widespread periodicity in quasars/blazars light curves up untill now. On the other hand, the search for supermassive binary black holes in extragalactic sources should become a major research topic in the next years.

As a final consideration, we point out that our 10-year observations probably mapped 2 distinct phases: a stage of low or intermediate optical luminosity (1994-2000) and a phase of mild flaring activity (2001). That dual and possibly cyclic scenario might well be confirmed by the behavior of the radio flux and structure during the same years (Gabuzda et al. 1994; Gómez et al. 2001; Agudo et al. 2006). The optical flux is believed to be mainly originated in the very inner regions of the jet, even if the stronger variability could occur much farther from the central engine than previously expected (Marscher 2005). Hence it is reasonable to conceive a correlation between the optical and radio flux on long-term scales, and during the most active events. An improved continuous and longer optical monitoring of PKS $0735+178$, and the comparison of the optical flare events with the ejection and evolution of the superluminal radio knots based on long-term data records, will allow us to shed light on the physics of the jet flow and flare mechanisms in this interesting object.

Acknowledgements. First of all we wish to warmly thank all the collaborators, observers, students, and technicians who contributed, in several occasions over the past years, to observations, technical support, data reduction, analysis, and discussions within the four teams. With the hope that we have not, unintentionally, forgotten anyone, we would like to express our gratitude to the following people. For the Perugia team: M. Bagaglia, M. Luciani, N. Marchili, S. Pascolini, V. Picarelli, N. Rizzi, F. Roncella, G. Sciuto, and C. Spogli. For the Torino team S. Bosio, M. Cavallone, M. Chiaberge, S. Crapanzano, G. De Francesco, G. Ghisellini, G. Latini, L. Ostorero, M. Puccio, and G. Sobrito. For the Tuorla team: R. Rekola. For the Sabadell team: J.M. Coloma, R. Costa, S. Esteva, E. Forné, and R. Ramajo.

Dr. Bochen Qian is to be thanked for providing most of the historical optical data. Dr. Jun-Hui Fan is to be thanked for general comments. Dr. Alex W. Fullerton is to be thanked for providing the CLEAN code. The anonymous referee is acknowledged because his detailed report and discussion helped to improve and clarify the paper. The groups of the Perugia, Torino, and Tuorla Observatories belonging to the Research Training Network ENIGMA, acknowledge the funding by the European Community's Human Potential Programme under contract HPRN-CT-2002-00321. The Perugia and Torino optical monitoring programs have been partly supported by the Italian Ministry for Instruction, University and Research (MIUR) under grant Cofin2001/028773. The Tuorla monitoring program has been partially supported by the Academy of Finland. This research has made use also of: the SIMBAD database (CDS, Strasbourg), the NASA/IPAC NED database (JPL CalTech and NASA), the HEASARC database (LHEA NASA/GSFC and SAO), and the Smithsonian/NASA's ADS bibliographic databases.

\section{References}

Agudo, I., Gómez, J. L., Gabuzda, D. C., et al. 2006, A\&A, 453, 477

Alexander, T. 1997, in Astronomical Time Series, ed. Maoz, Sternberg \& Leibowitz (Dordrecht: Kluwer), 163

Aller, M. F., Aller, H. D., Hughes, P. A., \& Latimer, G. E. 1999, ApJ, 512, 601

Bai, J. M., Xie, G. Z., Li, K. H., Zhang, X., \& Liu, W. W. 1999, A\&AS, 136, 455 Bååth, L. B., \& Zhang, F. J. 1991, A\&A, 243, 328

Bessell, M. S. 1979, PASP, 91, 589

Bessell, M. S. 1990, PASP, 102, 1181

Bessell, M. S. 2005, ARA\&A, 43, 293

Blake, G. M. 1970, Astrophys. Lett., 6, 201

Bregman, J. N., Glassgold, A. E., \& Huggins, P. J. 1981, ApJ, 249, 13
Bregman, J. N., Glassgold, A. E., Huggins, P. J., et al. 1984, ApJ, 276, 454 Brown, L. M. J., Robson, E. I., Gear, W. K., \& Smith, M. G. 1989, ApJ, 340, 150 Burbidge, G., \& Hewitt, A. 1987, AJ, 93, 1

Böttcher, M., Harvey, J., Joshi, M., et al. 2005, ApJ, 631, 169

Böttcher, M., \& Chiang, J. 2002, ApJ, 581, 127

Carswell, R. F., Strittmatter, P. A., Williams, R. E., Kinman, T. D., \& Serkowski, K. 1974, ApJ, 190, L101

Ciaramella, A., Bongardo, C., et al. 2004, A\&A, 419, 485

Ciprini, S., Tosti, G., Teräsranta, H., \& Aller, H. D. 2004, MNRAS, 348, 1379

Clements, S. D., Smith, A. G., Aller, H. D., \& Aller, M. F. 1995, AJ, 110, 529

Cotton, W. D., Wittels, J. J., Shapiro, I. I., et al. 1980, ApJ, 238, L123

Daubechies, I., 1992, Ten Lectures on Wavelets, Philadelphia: Soc. for Industrial \& Applied Math. (SIAM)

Deeming, T. J. 1975, Ap\&SS, 36, 137

Della Ceca, R., Palumbo, G. G. C., et al. 1990, ApJS, 72, 471

Edelson, R. A., \& Krolik, J. H. 1988, ApJ, 333, 646

Elvis, M., Plummer, D., Schachter, J., \& Fabbiano, G. 1992, ApJS, 80, 257

Falomo, R., \& Ulrich, M.-H. 2000, A\&A, 357, 91

Fan, J. H., \& Lin, R. G. 1999, ApJS, 121, 131

Fan, J. H., \& Lin, R. G. 2000, ApJ, 537, 101

Fan, J. H., Xie, G. Z., Lin, R. G., et al. 1997, A\&AS, 125, 525

Farge, M. 1992, Annu. Rev. Fluid Mech., 24, 395

Fiorucci, M., \& Munari, U. 2003, A\&A, 401, 781

Fiorucci, M., \& Tosti, G. 1996, A\&AS, 116, 403

Fiorucci, M., Tosti, G., \& Rizzi, N. 1998, PASP, 110, 105

Fiorucci, M., Ciprini, S., \& Tosti, G. 2004, A\&A, 419, 25

Foster, G. 1995, AJ, 109, 1889

Foster, G. 1996, AJ, 112, 1709

Gabuzda, D. C., Gómez, J. L., \& Agudo, I. 2001, MNRAS, 328, 719

Gabuzda, D. C., Wardle, J. F. C., Roberts, D. H., Aller, M. F., \& Aller, H. D. 1994, ApJ, 435, 128

Gabuzda, D. C., Wardle, J. F. C., \& Roberts, D. H. 1989, ApJ, 338, 743

Georganopoulos, M., \& Marscher, A. P. 1998, ApJ, 506, L11

Ghisellini, G., Villata, M., Raiteri, C. M., et al. 1997, A\&A, 327, 61

Ghosh, K. K., Ramsey, B. D., Sadun, A. C., \& Soundararajaperumal, S. 2000, ApJS, 127, 11

Gómez, J. L., Guirado, J. C., Agudo, I., et al. 2001, MNRAS, 328, 873

Gómez, J., Marscher, A. P., Alberdi, A., \& Gabuzda, D. C. 1999, ApJ, 519, 642

Gu, M. F., Lee, C.-U., Pak, S., Yim, H. S., \& Fletcher, A. B. 2006, A\&A, 450, 39

Hanski, M. T., Takalo, L. O., \& Valtaoja, E. 2002, A\&A, 394, 17

Hartman, R. C., Bertsch, D. L., Bloom, S. D., et al. 1999, ApJS, 123, 79

Homan, D. C., Ojha, R., Wardle, J. F. C., et al. P. A. 2002, ApJ, 568, 99

Horne, J. H., \& Baliunas, S. L. 1986, ApJ, 302, 757

Högbom, J. A. 1974, A\&AS, 15, 417

Hufnagel, B. R., \& Bregman, J. N. 1992, ApJ, 386, 473

Hughes, P. A., Aller, H. D., \& Aller, M. F. 1992, ApJ, 396, 469

Hutchings, J. B., Johnson, I., \& Pyke, R. 1988, ApJS, 66, 361

Jurkevich, I. 1971, Ap\&SS, 13, 154

Kaiser, G., 1994, A Friendly Guide to Wavelets (Boston: Birkhauser editions)

Katajainen, S., Takalo, L. O., Sillanpää, A., et. al 2000, A\&AS, 143, 357

Kataoka, J., Takahashi, T., Makino, F., et al. 2000, ApJ, 528, 243

Kellermann, K. I., Vermeulen, R. C., Zensus, J. A., \& Cohen, M. H. 1998, AJ, 115,1295

Kellermann, K. I., Lister, M. L., Homan, D. C., et al. 2004, ApJ, 609, 539

Kidger, M. R. 1989, A\&A, 226, 9

Kirk, J. G., Rieger, F. M., \& Mastichiadis, A. 1998, A\&A, 333, 452

Kubo, H., Takahashi, T., Madejski, G., et al. 1998, ApJ, 504, 693

Kühr, H., Witzel, A., Pauliny-Toth, I. I. K., \& Nauber, U. 1981, A\&A, 45, 367

Lafler, J., \& Kinman, T. D. 1965, ApJS, 11, 216

Lainela, M., \& Valtaoja, E. 1993, ApJ, 416, 485

Lähteenmäki, A., \& Valtaoja, E. 1999, ApJ, 521, 493

Lehto, H. J., \& Valtonen, M. J. 1996, ApJ, 460, 207

Lin, R. G., \& Fan, J. H. 1998, Ap\&SS, 259, 67

Lomb, N. R. 1976, Ap\&SS, 39, 447

Lu, P. K. 1972, AJ, 77, 829

Madejski, G. M., \& Schwartz, D. A. 1988, ApJ, 330, 776

Marscher, A. P. 1977, AJ, 82, 781

Marscher, A. 1980, Nature, 288, 12

Marscher, A. P. 2005, Mem. Soc. Astron. It., 76, 168

Massaro, E., \& Trevese, D. 1996, A\&A, 312, 810

Massaro, E., Nesci, R., Perola, G. C., Lorenzetti, D., \& Spinoglio, L. 1995, A\&A, 299, 339

Mead, A. R. G., Ballard, K. R., Brand, P. W. J. L., et al. 1990, A\&AS, 83, 183

McGimsey, B. Q., Williamon, R. M., \& Miller, H. R. 1976, AJ, 81, 750

Nilsson, K. et al. 2006, in preparation

Nolan, P. L., Tompkins, W. F., Grenier, I. A., \& Michelson, P. F. 2003, ApJ, 597, 615 
Ojha, R., Homan, D. C., Roberts, D. H., et al. 2004, ApJS, 150, 187

Ostorero, L., Villata, M., \& Raiteri, C. M. 2004, A\&A, 419, 913

Papadakis, I. E., \& Lawrence, A. 1993, MNRAS, 261, 612

Percival, D.B., \& Walden, A. T. 2002, Wavelet Methods for Time Series Analysis (Cambridge: Cambridge University Press)

Perlman, E. S., \& Stocke, J. T. 1994, AJ, 108, 56

Pollock, J. T., Pica, A. J., Smith, A. G., et al. 1979, AJ, 84, 1658

Pursimo, T., Nilsson, K., Sillanpää, A., Takalo, L. O., \& Heidt, J. 1999, BL Lac Phenomenon, ASP Conf. Ser. 159, 385

Pursimo, T., Nilsson, K., Takalo, L. O., et al. 2002, A\&A, 381, 810

Qian, B., \& Tao, J. 2004, PASP, 116, 161

Raiteri, C. M., Villata, M., Tosti, G., et al. 2003, A\&A, 402, 151

Ravasio, M., Tagliaferri, G., Ghisellini, G., \& Tavecchio, F. 2004, A\&A, 424, 841

Rector, T. A., \& Stocke, J. T. 2001, AJ, 122, 565

Rieger, F. M., \& Mannheim, K. 2000, A\&A, 359, 948

Roberts, D. H., Lehar, J., \& Dreher, J. W. 1987, AJ, 93, 968

Rutman, J. 1978, Proc. IEEE, 66, 1048

Sagar, R., Stalin, C. S., Gopal-Krishna, G., \& Wiita, P. J. 2004, MNRAS, 348, 176

Scargle, J. D. 1982, ApJ, 263, 835

Scarpa, R., Urry, C. M., Falomo, R., Pesce, J. E., \& Treves, A. 2000, ApJ, 532, 740

Schlegel, D. J., Finkbeiner, D. P., Davis, M. 1998, ApJ, 500, 525

Sillanpää, A., Takalo, L. O., Pursimo, T., et al. 1996, A\&A, 315, L13

Simonetti, J. H., Cordes, J. M., \& Heeschen, D. S. 1985, ApJ, 296, 46

Sitko, M. L., \& Sitko, A. K. 1991, PASP, 103, 160

Smith, A. G., \& Nair, A. D. 1995, PASP, 107, 863

Smith, A. G., Leacock, R. J., \& Webb, J. R. 1988, in LNP Vol. 307: Active Galactic Nuclei, ed. by H. R. Miller \& P. J. Wiita (Berlin: Springer-Verlag), 158

Smith, A. G., Nair, A. D., Leacock, R. J., \& Clements, S. D. 1993, AJ, 105, 437
Smith, P. S., Balonek, T. J., Heckert, P. A., Elston, R., \& Schmidt, G. D. 1985, AJ, 90, 1184

Smith, P. S., Balonek, T. J., Elston, R., \& Heckert, P. A. 1987, ApJS, 64, 459

Stellingwerf, R. F. 1978, ApJ, 224, 953

Stickel, M., Fried, J. W., \& Kuehr, H. 1993, A\&AS, 98, 393

Takalo, L. O. 1991, A\&AS, 90, 161

Takalo, L. O., Sillanpää, A., Nilsson, K., Kidger, M., de Diego, J. A., \& Piirola, V. 1992, A\&AS, 94, 37

Teräsranta, H., Tornikoski, M., Valtaoja, E., et al. 1992, A\&AS, 94, 121

Teräsranta, H., Achren, J., Hanski, M., et al. 2004, A\&A, 427, 769

Tommasi, L., Palazzi, E., Pian, E., et al. 2001, A\&A, 376, 51

Tornikoski, M., Valtaoja, E., Teräsranta, H., et al. 1994, A\&A, 289, 673

Tosti, G., Pascolini, S., \& Fiorucci, M. 1996, PASP, 108, 706

Valtaoja, L., Sillanpää, A., Valtaoja, E., Shakhovskoi, N. M., \& Efimov, I. S. 1991, AJ, 101, 78

Valtaoja, L., Karttunen, H., Valtaoja, E., Shakhovskoy, N. M., \& Efimov, Y. S. 1993, A\&A, 273, 393

Valtaoja, E., Teräsranta, H., Tornikoski, M., et al. 2000, ApJ, 531, 744

Veron, P., \& Veron, M. P. 1975, A\&A, 39, 281

Villata, M., Raiteri, C. M., Sillanpaa, A., \& Takalo, L. O. 1998, MNRAS, 293, L13

Villata, M., Raiteri, C. M., Kurtanidze, O. M., et al. 2002, A\&A, 390, 407

Villata, M., Raiteri, C. M., Kurtanidze, O. M., et al. 2004, A\&A, 421, 103

Vio, R., Kristensen, N. R., Madsen, H., \& Wamsteker, W. 2005, A\&A, 435, 773

Voges, W., Aschenbach, B., Boller, Th., et al. 1999, A\&A, 349, 389

Wagner, S. J. 1996, A\&AS, 120, 495

Webb, J. R., Smith, A. G., Leacock, R. J., et al. 1988, AJ, 95, 374

Wing, R. F. 1973, AJ, 78, 684

Xie, G. Z., Li, K. H., et al. 1992, ApJS, 80, 683

Zhang, L. Z., Fan, J.-H., \& Cheng, K.-S. 2002, PASJ, 54, 159

Zhang, X., Zhang, L., Zhao, G., et al. 2004, AJ, 128, 1929

Zekl, H., Klare, G., \& Appenzeller, I. 1981, A\&A, 103, 342 